Research Articles: Cellular/Molecular

\title{
Deconstructing Synaptotagmin-1's Distinct Roles in Synaptic Vesicle Priming and Neurotransmitter Release
}

https://doi.org/10.1523/JNEUROSCI.1945-21.2022

Cite as: J. Neurosci 2022; 10.1523/JNEUROSCI.1945-21.2022

Received: 27 September 2021

Revised: 18 January 2022

Accepted: 23 January 2022

This Early Release article has been peer-reviewed and accepted, but has not been through the composition and copyediting processes. The final version may differ slightly in style or formatting and will contain links to any extended data.

Alerts: Sign up at www.jneurosci.org/alerts to receive customized email alerts when the fully formatted version of this article is published.

Copyright (c) 2022 Bouazza-Arostegui et al.

This is an open-access article distributed under the terms of the Creative Commons Attribution 4.0 International license, which permits unrestricted use, distribution and reproduction in any medium provided that the original work is properly attributed. 
4 Abbreviated title: Re-analysis of Synaptotagmin-1 synaptic functions.

5

6 Boris Bouazza-Arostegui ${ }^{1,2}$, Marcial Camacho ${ }^{1,2}$, Marisa M. Brockmann ${ }^{1,2}$, Sina Zobel ${ }^{1,2}$ and Christian 7 Rosenmund ${ }^{1,2}$

$8{ }^{1}$ Charité - Universitätsmedizin Berlin, corporate member of Freie Universität Berlin and Humboldt-Universität zu 9 Berlin, Institute of Neurophysiology, 10117, Berlin, Germany.

$10{ }^{2}$ NeuroCure Cluster of Excellence, 10117, Berlin, Germany.

12 Corresponding author: Correspondence should be addressed to Christian Rosenmund,

13 christian.rosenmund@charite.de

15 Number of pages: 28 , Number of figures: 8 , Number of Tables: 1 , Number of extended data: 1 , Number of words

16 for Abstract: 181, Number of words for introduction: 611, Number of words for discussion: 1487

Conflict of interest: The authors declare no competing financial interest.

Acknowledgements: This work was supported by grants from the German Research Council (CRC 958 and Reinhart Koselleck project). Boris Bouazza-Arostegui was supported by the NeuroCure Cluster of Excellence with a NeuroCure PhD Fellowship. We are grateful to Berit Söhl-Kielczynski, Bettina Brokowski, Katja Pötschke, Sabine Lenz and Heike Lerch for excellent technical support and to Dr. Melissa Herman for her comments and helpful discussion. We thank the services of the Charite Viral core facility for virus production and

27 characterization. We also thank the services of Advanced Medical Bioimaging Core Facility (AMBIO), Nikon 28 imaging core facilities at the Charite Campus Mitte (Berlin, Germany). M.C. current address: Unidad de 29 Farmacología, Facultad de Medicina, Universidad de La Laguna, 38200 La Laguna, Tenerife, Spain. M.M.B.

30 current address: Center of Molecular Neurobiology Hamburg, Hamburg 2051, Germany. 


\section{Abstract}

32 Synaptotagmin-1 (SYT1) is a synaptic vesicle resident protein that interacts via its C2 domain with 33 anionic lipids from the plasma membrane (PM) in a calcium-dependent manner to efficiently trigger 34 rapid neurotransmitter (NT) release. In addition, SYT1 acts as a negative regulator of spontaneous 35 NT-release and regulates synaptic vesicle (SV) priming. How these functions relate to each other 36 mechanistically and what role other synaptotagmin (SYT) isoforms play in supporting and 37 complementing the role of SYT1 is still under intensive investigation. In this work, we analyzed 38 SYT1's three putative functions in exocytosis by systematically varying its expression in autaptic 39 hippocampal glutamatergic neurons from mice of either sex. We find that regulation of release 40 probability is most sensitive to variation of expression levels, while its impact on vesicle priming is 41 least sensitive. Also, loss of SYT1 phenotypes on spontaneous release and vesicle priming is 42 compensated in less mature synaptic cultures by redundant support from SYT7. Overall, our data 43 help resolving some controversies in SYT1 functions in exocytosis and in our understanding of how 44 SYT1 contributes to the pathophysiology underlying SYT1 related human neurologic disorders.

\section{Significance statement}

46 Our work clarifies the functions of Synaptotagmin-1 (SYT1) protein in synaptic vesicle priming, 47 spontaneous and calcium-evoked neurotransmitter release and analyze whether these occur at 48 different stages of synaptic responses by examining their relative sensitivity to protein concentration 49 at the synaptic terminal. We demonstrate that these synaptic functions are unequally sensitive to both 50 protein levels and neuronal stage, indicating that they operate under distinct molecular mechanisms. 51 Furthermore, we analyze how these functions are modulated by another synaptotagmin isoform 52 expression. We show that to understand the phenotype displayed by SYT1 knockout neurons $\left(\right.$ Syt $\left.{ }^{-1 /}\right)$ 53 is necessary to consider the interplay between SYT1 and SYT7 molecules at the presynaptic terminal.

\section{Introduction}

55 Calcium evoked neurotransmitter (NT) release from the presynaptic nerve terminal occurs in less than 56 one millisecond following the activation of voltage-gated calcium channels triggered by the arrival of 57 an action potential (AP) (Katz, 1969; Sabatini and Regehr, 1999). A key element in orchestrating the 58 rapid and efficient presynaptic $\mathrm{Ca}^{2+}$-evoked NT release is the synaptic vesicular protein 
Synaptotagmin-1 (SYT1) (DiAntonio and Schwarz, 1994; Fernández-Chacón et al., 2001; Rizo and

$60 \mathrm{Xu}, 2015)$. Biochemical studies have demonstrated that SYT1 is a phospholipid-binding machine that

61 acts in a calcium-dependent manner via its $\mathrm{C} 2 \mathrm{~A}$ and $\mathrm{C} 2 \mathrm{~B}$ cytoplasmic domains to trigger NT release

62 (Perin et al., 1990; Brose et al., 1992; Sutton et al., 1995; Chapman and Davis, 1998; Fernandez et 63 al., 2001).

64 In Drosophila and mammalian synapses in the absence of Syt1, AP-evoked synchronous release is drastically impaired (Broadie et al., 1994; Geppert et al., 1994; Yoshihara and Littleton, 2002; Nishiki and Augustine, 2004; Maximov and Südhof, 2005; Xue et al., 2008), but structure/function analysis of the C2B domain reveals that SYT1 is involved in two additional functions. SYT1 also acts as a "clamping factor" suppressing spontaneous release of synaptic vesicles (SVs) (DiAntonio and Schwarz, 1994; Littleton et al., 1994; Chicka et al., 2008; Xu et al., 2009) and different research groups have shown a role for SYT1 in SV docking/priming, mediating the recruitment of vesicles to the plasma membrane (PM) by the interaction with phosphatidylserine (PS) and phosphatidylinositol 4, 5-bisphosphate (PIP2) (Reist et al., 1998; Liu et al., 2009; Bacaj et al., 2015; Chang et al., 2018). However, how these functions of SYT1 mechanistically interact is currently unclear.

In addition to the different roles of SYT1 in the process of neurotransmitter release, other synaptotagmin isoforms have been implicated in distinct and overlapping roles. For example, the Synaptotagmin-7 (SYT7) isoform has been claimed to be a high-affinity calcium sensor for asynchronous release (Bacaj et al., 2013; Chen and Jonas, 2017), a regulator of short term plasticity (STP) (Wen et al., 2010; Jackman et al., 2016; Chen et al., 2017b; Fujii et al., 2021) and to act redundantly with SYT1 in the maintenance of the ready releasable pool (RRP) of SVs (Bacaj et al., 2015). Furthermore, the expression of synaptotagmin isoforms is regulated in a cell type specific manner. At the calyx of Held synapse and in some GABAergic neurons, the vesicular Synaptotagmin2 (SYT2) protein, the closest relative isoform of SYT1 (Geppert et al., 1991; Marquèze et al., 1995), regulates neurotransmission redundantly with SYT1 (Pang et al., 2006; Chen et al., 2017a). Synaptic transmission depends on SYT1 in the early postnatal calyx of Held synapses but later switches to SYT2 (Kochubey et al., 2016), suggesting that dynamic changes in synaptotagmin content at synapses during development and their redundant functions in priming and release have an impact in synaptic function. Consistent with this idea, heterozygotic de novo mutations in the syt1 gene 
detected in patients have been shown to be associated with a neurodevelopmental disorder (Baker et al., 2015, 2018; Bradberry et al., 2020).

In this study, we therefore aim to analyze the proposed distinct roles of SYT1 in vesicle

92 priming, spontaneous and evoked release and investigate these functions in context of development,

93 its interplay with SYT7 and the relative contribution of SYT1 expression levels on these functions. We

94 found that the loss of function phenotype of SYT1 depends on the maturation stage of neurons, where immature neurons can compensate the loss of SYT1 with expression of SYT7 in 2 of the 3 SYT1 functions. Furthermore, by systematically investigating the three key functions of SYT1 as a function of protein levels we define distinct sensitivity of these processes arguing a distinct role of SYT1 in these different functions.

99 Materials and Methods

\section{$100 \quad$ Animals}

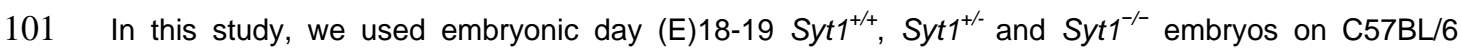

102 background of either sex generated by interbreeding Syt1 heterozygous mice as described previously 103 (Xue et al., 2008). All procedures and animals used were handled according to the regulations of the 104 Directive 2010/63/EU of the European Parliament on the protection of animals used for scientific 105 purposes and approved by the Berlin state authorities under the license number G-Project 106/20 and 106 the animal welfare committee of Charité - Universitätsmedizin Berlin.

\section{Lentiviral constructs and production}

108 Lentiviruses were generated by the Viral Core Facility of the Charité - Universitätsmedizin Berlin. All 109 lentiviral particles were produced as described in previous work (Lois et al., 2002). Lentiviral Syt1 110 construct used for rescue and overexpression experiments was generated from mouse Syt1 cDNA 111 (NCBI reference sequence: NM_001252341). The cDNA was cloned into a lentiviral vector (FUGW) 112 with a human synapsin1 promoter and following a nuclear localization signal (NLS)-RFP-P2A 113 expression cassette for identification of infected cells. A lentivirus expressing only NLS-RFP-P2A 114 controlled by the human synapsin1 promoter served as control. For shRNA mediated knockdown 115 experiments of SYT1 protein, an shRNA cassette containing a 19bp target sequence of Syt1 116 (5'AGTCTTCCTGCTGCCCGAC-3') was cloned downstream of a U6 promoter that also contained a 117 ubiquitin promoter driven RFP expression cassette ( $f(U 6)$ shRNA-Syt1.Ubi-RFP.WPRE). For shRNA 118 mediated knockdown of SYT7 a 21bp targeting sequence KD607 from (Bacaj et al., 2013) was used 
119 under the control of U6 promoter expression cassette within a lentivirus that also contained a human

120 synapsin1 promoter to control the nuclear RFP expression cassette (f(U6) shRNA-Syt7.Syn-NLS-

121 RFP.WPRE). A scramble RNA (scRNA) sequence served in both shRNA constructs as control

122 (Watanabe et al., 2014). The lentiviral titer for all generated constructs was estimated by

123 quantification of hippocampal neurons in mass culture expressing a fluorescent marker after DIV7.

\section{Autaptic neuronal culture and viral infection}

125 For electrophysiology recordings and immunocytochemistry experiments autaptic glutamatergic 126 hippocampal neurons were prepared as previously reported by (Bekkers and Stevens, 1991). Briefly, 127 hippocampal neurons derived from Syt1 $1^{+/+}$, Syt $1^{+/}$and Syt $1^{-/-}$embryonic mice (E18-19) of either sex 128 were used and plated at a density of 300 cells $/ \mathrm{cm}^{2}$ on $30 \mathrm{~mm}$ coverslips containing astrocytic 129 microislands. Astrocytes were obtained from cerebral cortices of postnatal day (P) 0-2 C57BL/6N mice and plated at a density of $5000 \mathrm{cells} / \mathrm{cm}^{2}$ on the micropattern coverslips 1 week before of the preparation of the neurons. Autaptic neuronal cultures were infected with the appropriate viral

\section{Electrophysiology}

134 Whole-cell patch-clamp recordings in autaptic neurons were performed at 11-21 days in vitro (DIV).

135 All electrophysiological recordings were done at room temperature with a Multiclamp 700B amplifier 136 (Molecular Devices) controlled by Clampex 10 software (Molecular Devices). Data were digitally 137 sampled at $10 \mathrm{kHz}$ and were filtered using a low-pass Bessel filter at $3 \mathrm{kHz}$. Series resistance was 138 compensated up to $70 \%$. Autaptic cultures during recordings were immersed in an extracellular 139 solution that contained the following (in $\mathrm{mM}$ ): $140 \mathrm{NaCl}, 2.4 \mathrm{KCl}, 10 \mathrm{HEPES}, 10$ glucose, $2 \mathrm{CaCl}_{2}$ and $1404 \mathrm{MgCl}_{2}$. Borosilicate glass patch pipettes were pulled using a multistep puller ( $\mathrm{P}-1000$, Sutter 141 Instruments). Pipettes with resistance (3-5 MOhms) were filled with $\mathrm{KCl}$-based intracellular solution 142 containing (in mM): $136 \mathrm{KCl}, 17.8$ HEPES, 1 EGTA, $4.6 \mathrm{MgCl}_{2}, 4$ ATP-Na ${ }_{2}, 0.3$ GTP-Na, 12 creatine 143 phosphate and $50 \mathrm{U} / \mathrm{ml}$ phosphocreatine kinase. Both internal and extracellular solutions were 144 adjusted to $300 \mathrm{mOsm}$ and $\mathrm{pH}$ 7.4. Neurons were clamp at $-70 \mathrm{mV}$ during recordings. Exclusion 145 criteria were stablished for patched cells with a leak current higher than -200 pA. Single APs were 146 evoked by a $2 \mathrm{~ms}$ depolarization step to $0 \mathrm{mV}$ and excitatory postsynaptic currents (EPSCs) were

147 recorded. To measure the synchronicity of synaptic responses we inverted the EPSC charge and 148 integrate the signal. Then, we fitted a two components function provided by Axograph X (Axograph 
Scientific), from which we calculated their relative contribution to the total charge released. The first

150 component represents the fast and synchronous release part of the EPSC charge and the second component the slow and asynchronous release part of the EPSC charge. s of external solution containing $500 \mathrm{mM}$ sucrose (Rosenmund and Stevens, 1996). Evoked-sucrose currents were recorded and the RRP size were estimated by integrating the area of the evokedsucrose current with the steady-state current set as the baseline. Vesicular release probability (Pvr) was calculated as the ratio between the charge of the EPSC and the evoked-sucrose charge.

We calculated the paired-pulsed ratio (PPR) by dividing the second EPSC amplitude (EPSC2) by the first (EPSC1). A train of AP stimulation at $20 \mathrm{~Hz}$ for $5 \mathrm{~s}$ was recorded to assess short term plasticity. The synaptic responses from the train of AP were normalized to the first EPSC peak amplitude.

To analyze mini excitatory postsynaptic currents (mEPSC), electrophysiological traces were

162 filtered at $1 \mathrm{kHz}$ and the range of parameters for inclusion of selected events using a conventionally 163 defined template algorithm in Axograph X (Axograph Scientific) were 5-200 pA, 0.15-1.5 ms rise-time 164 and 0.5-5 ms half-width. False-positive events were excluded by subtracting events detected from 165 traces in the presence of AMPA receptor antagonist, NBQX $(3 \mu \mathrm{M})$. Spontaneous release rate was calculated by dividing the mEPSC frequency by the number of synaptic vesicles in the RRP. The number of synaptic vesicles in the RRP was calculated by dividing the RRP size by the mEPSC charge. Off-line analysis was performed using Axograph X (Axograph Scientific).

170 Autaptic hippocampal neurons were immunostained as previously reported by (Xue et al., 2007). 171 Briefly, neurons were fixed with 4\% paraformaldehyde (PFA; Sigma-Aldrich) for 10 min at 11 and 16 172 DIV for Figure 3 and 15-21 DIV for Figures 2, 5, 6, 7 and 8. Primary antibodies monoclonal mouse 173 anti-Synaptotagmin-1 (1:1000; Synaptic System), polyclonal guinea pig anti-VGLUT1 (1:4000; 174 Synaptic System) and polyclonal rabbit anti-Synaptogamin 7 (1:500; Synaptic System) were used. 175 Secondary antibodies (1:500) conjugated with Alexa Fluor 405, 488 or 647 (Jackson 176 ImmunoResearch) were utilized to visualize fluorescence. For quantitative assessment, all groups 177 compared in one experiment were processed in parallel using identical antibodies solutions and other 178 reagents. 
Single neurons on the astrocytic micro-islands were imaged by using a Nikon Scanning

180 Confocal A1Rsi+ with a 60x, 1.4 NA oil-immersion objective. For acquiring all the images, we used

181 the same microscope and camera with identical acquisition settings for all experimental groups.

182 Overexposure and photobleaching was avoided by checking the fluorescence signal saturation in 183 synaptic boutons. $Z$ stacks of neurons were set with a $0.3 \mu \mathrm{m}$ inter-stack interval and total $\mathrm{z}$ axis 184 range of 5-6 $\mu \mathrm{m}$, and a sum of intensity projection was further used for analyses. The images were 185 obtained at $1024 \times 1024$ pixels resolution and at the pixel size of $0,2 \mu \mathrm{m}$. During acquisition, laser 186 settings were applied identically to all coverslips quantified in all experimental groups compared.

187 The analysis was performed using ImageJ software (US National Institutes of Health) by 188 drawing ROIs of 50 synapses per neuron. Excitatory synapses (region of interest, ROI) were defined 189 by staining for the synaptic vesicle (SV) marker VGLUT1. SYT1 or SYT7 fluorescence intensity signal 190 for each synapse was measured within the defined ROI. Five to ten autaptic neurons were collected 191 per condition per culture and at least three independent cultures were analyzed per experiment. 192 Relative expression level of SYT1 among groups was calculated by normalizing the measured 193 intensities of SYT1 to that of VGLUT1. The data were normalized to the control of each experiment.

\section{Experimental design and statistics}

195 For electrophysiology and immunocytochemistry experiments, we recorded and imaged 196 approximately the same number of autaptic neurons from each experimental group each day to reduce data variability. For each parameter analyzed, the number of neurons used ( $\mathrm{n}$ number) and

198 the number of independent cultures $(\mathrm{N})$ is indicated in the figure, specifically within the bar graph $199(\mathrm{n} / \mathrm{N})$. Data were acquired from at least three independent hippocampal autaptic cultures generated 200 from three different animals $(\mathrm{N}=3)$ to minimize culture-culture variation. For Figure 7 we chose the nonlinear regression model standard Hill equation. We did not constrain any parameter of the model to a constant value. We performed a global nonlinear regression; we specify that parameters are shared to fit all data sets. All data points are weighted equally in the model.

Data from each experimental group were pooled together except for Figure 2, 7 and 8 which were normalized to the mean value of the control group. Statistical analyses were performed using Prism 8 (GraphPad). All data were first subjected to Pearson omnibus K2 normality testing. Two-tailed unpaired t test or one-way ANOVA test for normally distributed data and Mann-Whitney test or 
comparison methods were used following ANOVA test. Significance and $p$ values were calculated and

210 are shown in the corresponding Figure or Table.

\section{Results}

212 Phenotype of Synaptotagmin-1 loss in synaptic vesicle priming and spontaneous release is 213 sensitive to neuronal maturation state

214 Given the controversy over SYT1 involvement in clamping and priming SVs, the observation of 215 developmentally dependent effects of SYT2 in the calyx of Held (Kochubey et al., 2016) and the 216 discovery of new point mutations in SYT1 gene associated with a neurodevelopmental disorder 217 (Baker et al., 2018), we aimed to re-analyze the roles of Synaptotagmin-1 in SV priming, 218 synchronized and spontaneous release as a function of neuronal culture age. We recorded from 219 autaptic hippocampal neurons derived from Syt1 $1^{+/+}$or $S y t 1^{-/}$mice across a ten-day time period (11-21 220 days in vitro (DIV)), and subsequently grouped our results into three different classes (11-12, 15-16 221 and 20-21 DIV).

To assess SV priming, we measured the readily releasable pool (RRP) of SVs using $5 \mathrm{~s}$ application of 500 mOsm hypertonic solution (Rosenmund and Stevens, 1996) and integrated the 224 transient component of the evoked inward current. While the RRP charge of Syt1 $1^{+/+}$and Syt1 $1^{-/}$ 225 autaptic neurons at DIV11-12 was indistinguishable, at both later culturing stages (DIV15-16) and 226 (DIV20-21) we detected significant $\sim 40-50 \%$ reduction in RRP charge in the Syt ${ }^{-/}$group (Figure 1A;

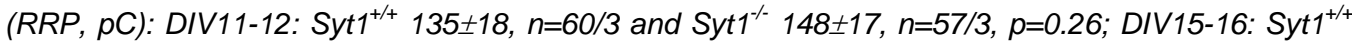
$477 \pm 54, n=58 / 3$ and Syt1 $247 \pm 33, n=47 / 3, p=0.0004 ;$ DIV20-21: Syt $1^{+/+} 759 \pm 89, n=44 / 3$ and Syt1

$229360 \pm 54, n=44 / 3, p<0.0001$; Mann-Whitney test). Next, we analyzed spontaneous neurotransmitter 230 release. We observed that loss of SYT1 led to no significant changes in spontaneous release rates for 231 the early DIV group while spontaneous release rate was enhanced in the two older DIV groups

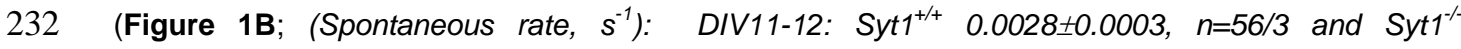
$233 \quad 0.0034 \pm 0.0003, \quad n=48 / 3, \quad p=0.0544 ; \quad D I V 15-16: \quad S y t 1^{+/+} \quad 0.0029 \pm 0.0005, \quad n=42 / 3$ and Syt1

$234 \quad 0.0065 \pm 0.0009, \quad n=43 / 3, \quad p<0.0001 ; \quad$ DIV20-21: Syt $1^{+/+} \quad 0.0019 \pm 0.0004, \quad n=44 / 3$ and Syt1 $2350.0052 \pm 0.0012, n=44 / 3, p<0.0001$; Mann-Whitney test), emphasizing that the impact of SYT1 loss on 236 spontaneous release, like the impact on RRP size, is dependent on the maturation state of the 237 neuron. 
238 In contrast, $\mathrm{Ca}^{2+}$-evoked release was massively affected regardless of culture age as indicated by the 239 reduction in EPSC amplitude and charge in all three DIV groups (Figure 1C; (EPSC amplitude, $n A$ ): 240 DIV11-12: Syt1 ${ }^{+/+}-1.17 \pm 0.19, n=60 / 3$ and Syt1 $1^{-/}-0.069 \pm 0.007, n=58 / 3, p<0.0001 ;$ DIV15-16: Syt1 ${ }^{+/+}$$2413.2 \pm 0.5, n=47 / 3$ and Syt $1^{-/}-0.097 \pm 0.012, n=43 / 3, p<0.0001 ;$ DIV20-21: Syt $1^{+/+}-4.19 \pm 0.58, n=44 / 3$ 242 and Syt1 $1^{-1}-0.085 \pm 0.012, n=44 / 3, p<0.0001$; Mann-Whitney test). Moreover, the vesicular release 243 probability (Pvr), as calculated by dividing the EPSC charge by the RRP charge, was similarly 244 reduced by $\sim 60 \%$ in all three DIV groups measured (Figure 1D; (Pvr, \%): DIV11-12: Syt1 ${ }^{+/+} 7.7 \pm 0.7$, $n=43 / 3, p<0.0001 ;$ DIV20-21: Syt $1^{+/+} 6.7 \pm 0.7, n=44 / 3$ and Syt $1^{-/} 2.3 \pm 0.3, n=44 / 3, p<0.0001$; MannWhitney test).

Lentiviral mediated SYT1 rescue experiments showed fully restored SV priming, and spontaneous and AP induced release efficacy in stages DIV15-21 (Figure 2; (RRP norm.): Syt1 ${ }^{+1+}$ $1 \pm 0.11, n=39 / 3$, Syt $1^{-1} 0.40 \pm 0.05, n=31 / 3, p<0.001$ and Syt $1_{+S Y T 1}^{-1} 0.93 \pm 0.15, n=29 / 3, p=n s /<0.05$;

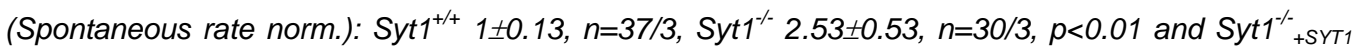
$0.96 \pm 0.12, n=26 / 3, p=n s /<0.05$; (EPSC charge norm.): Syt $1^{+/+} 1 \pm 0.16, n=42 / 3$, Syt1 $1^{-1} 0.34 \pm 0.05$, $n=34 / 3, p<0.01$ and Syt $1^{1 /}{ }_{+S Y T 1} 1.05 \pm 0.2, n=29 / 3, p=n s /<0.01$; (Pvr norm.): Syt $1^{+/+} 1 \pm 0.10, n=37 / 3$,

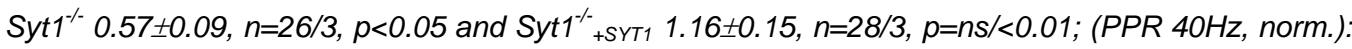
Syt1 $^{+/+} 1 \pm 0.06, n=32 / 3, \quad S y t 1^{-/} 2.15 \pm 0.20, n=29 / 3, p<0.0001$ and Syt $1_{+S Y T 1}^{-/} 0.97 \pm 0.07, n=24 / 3$, $p=n s /<0.0001$; Kruskal-Wallis test), indicating that the loss of function phenotype of SYT1 at older cultures was due to the loss of the protein and not due to developmental processes. Expression levels of exogenously expressed SYT1 were near wildtype levels as indicated by relative SYT1 immunofluorescence intensity from VGLUT1 marker positive compartments (Figure 2A and B;

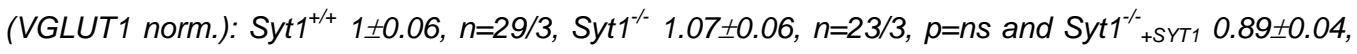
$n=26 / 3, p=n s / n s ;$ (SYT1/VGLUT1 norm.): Syt1 $1^{+/+} 1 \pm 0.05, n=29 / 3$, Syt1 $1^{-/} 0.16 \pm 0.01, n=23 / 3, p<0.0001$ and Syt1 ${ }_{+S Y T 1} 1.02 \pm 0.05, n=26 / 3, p=n s /<0.0001 ;$ Kruskal-Wallis test).

Overall, our data confirm that loss of SYT1 leads to loss of synchronized and efficient AP triggered release, but also that the impairment of RRP and clamping of spontaneous release only appears in more mature neurons. One possible explanation could be that the loss of SYT1 at early maturation stages may be compensated by other synaptotagmin isoform, which would be 
downregulated over neuronal development as similarly shown at the calyx of Held synapses

268 (Kochubey et al., 2016). These findings may provide an explanation for findings in autaptic

269 glutamatergic neurons where no significant changes in RRP size were reported when recordings were

270 pooled over extended times in vitro (e.g., Liu et al. 2009 DIV12-17). We therefore limited in the

271 remaining part of the experiments our experiments to neurons that were DIV15 or older.

272 Partial functional redundancy between Synaptotagmin-1 and Synaptotagmin-7

273 Recent genetic and functional analysis indicates that SYT7 plays a redundant role in SV priming 274 function (Bacaj et al., 2015). Moreover, the expression of the Synaptotagmin1/2 paralogs are 275 developmentally regulated at a murine auditory synapse, contributing to maturation dependent 276 variation in synaptic phenotypes in synaptotagmin loss of function mouse models (Kochubey et al., 277 2016). We therefore explored whether SYT7 expression in less mature hippocampal neurons 278 contributes to the milder phenotype of SYT1 loss by knocking-down SYT7 protein expression in Syt1 279 neurons. First, we examined SYT1 and SYT7 protein levels in presynaptic compartments at early 280 (DIV11-12) or intermediate stages (DIV15-16) in Syt1 ${ }^{+/+}$and Syt1 $1^{-/}$neurons and subsequently 281 measured at both stages the presynaptic SYT7 reduction affected by lentiviral expression of a SYT7 282 shRNA construct in Syt1 ${ }^{-1}$ autaptic neurons (Figure 3). We utilized VGLUT1 protein expression to 283 spatially define presynaptic compartments and to serve as reference signal for SYT1 and SYT7 284 expression levels. In Syt1 ${ }^{+/ t}$ neurons, SYT1 protein levels gradually increased with time of DIV but 285 stayed relatively constant in comparison to VGLUT1. In contrast, SYT7 protein expression levels 286 remained constant and, consequently, SYT7/VGLUT1 signal decreased over time in culture (Figure 287 3A and B; (VGLUT1 norm.): Syt $1^{+/+}{ }_{D I V 11} 1 \pm 0.12, n=21 / 3$ and Syt1 ${ }^{+/+}{ }_{D / V 16} 3.2 \pm 0.4, n=21 / 3, p<0.0001$; 288 Two-tailed unpaired t test; (SYT1 norm.): Syt $1^{+/+}{ }_{D I V 11} 1 \pm 0.10, n=21 / 3$ and Syt ${ }^{+/+}{ }_{D I V 16} 2.9 \pm 0.4, n=21 / 3$, 289 p<0.0001; Two-tailed unpaired t test; (SYT7 norm.): Syt ${ }^{+/+}{ }_{D I V 11} 1 \pm 0.14, n=21 / 3$ and $S y t 1^{+/+}{ }_{D I V 16}$ 290 1.3 $\pm 0.2, n=21 / 3, \quad p=n s ;$ Mann-Whitney test; (VGLUT1 norm.): Syt1 ${ }_{D / V 11}^{-1} 1 \pm 0.12, n=27 / 3$ and 291 Syt ${ }^{++t}{ }_{D I V 16} 2.3 \pm 0.36, n=28 / 3, p<0.001$; Mann-Whitney test). We tested how effective the SYT7 shRNA 292 reduced the presynaptic SYT7 protein expression and found an average reduction of app. $75 \%$ 293 across all DIVs tested using Syt ${ }^{-1 /}$ autaptic neurons (Figure 3C; (VGLUT1 norm.): Syt $1^{1-}{ }_{+R N A i(S y t 7) D I V 11}$ $2941 \pm 0.14, n=21 / 3$ and Syt $1^{-1}{ }_{+R N A i(S y t) D I V 16} 3.5 \pm 0.47, n=17 / 3, p<0.0001$; Mann-Whitney test; (SYT7DIV11 295 norm.): Syt $1^{-1} 1 \pm 0.21, n=27 / 3$ and Syt $1^{1 /}{ }_{+R N A(S y t 7)} 0.30 \pm 0.15, n=21 / 3, p<0.001$; Mann-Whitney test; 
296

(SYT7DIV16 norm.): Syt1 $1 \pm 0.13, n=28 / 3$ and Syt1 ${ }_{+R N A i(S y t 7)} 0.34 \pm 0.09, n=17 / 3, p<0.001 ;$ MannWhitney test; (SYT1DIV11 norm.): Syt1 ${ }^{+/+} 1 \pm 0.20, n=21 / 3$, Syt1 ${ }^{-/} 0.08 \pm 0.04, n=27 / 3, p<0.0001$ and Syt $1^{-1}{ }_{+ \text {RNAi(Syt7) }} 0.1 \pm 0.096, n=21 / 3, p<0.0001$; Kruskal-Wallis test; (SYT1DIV16 norm.): Syt $1^{+/+} 1 \pm 0.096$, $n=21 / 3, \quad$ Syt $1^{-1} 0.11 \pm 0.02, n=28 / 3, \quad p<0.0001$ and Syt ${ }_{+R N A i(S y t 7)} 0.07 \pm 0.06, n=17 / 3, \quad p<0.0001$; Kruskal-Wallis test).

Reduction of SYT7 protein expression in Syt1 ${ }^{-1}$ autaptic neurons reduced RRP size and increased mEPSC rates at 11-12 DIV and 15-16 DIV (Figure 4A and B; (RRP, pC): DIV11-12: Syt1 $1^{++}$

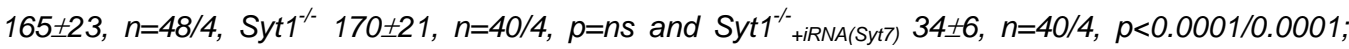

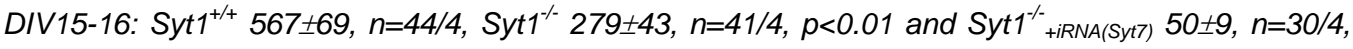
$p<0.0001 / 0.0001$; Kruskal-Wallis test; (Spontaneous rate, $\left.s^{-1}\right)$ : DIV11-12: Syt1 ${ }^{1++} 0.003 \pm 0.0004$, $n=48 / 4, \quad$ Syt1 ${ }^{-1} \quad 0.0043 \pm 0.0005, \quad n=33 / 4, \quad p=n s$ and Syt1 ${ }_{+i R N A(S y t 7)} \quad 0.012 \pm 0.0015, \quad n=38 / 4$, $p<0.0001 / 0.01 ;$ DIV15-16: Syt1 ${ }^{+/+} 0.0016 \pm 0.0002, n=44 / 4$, Syt1 $1^{-/} 0.0054 \pm 0.0012, n=41 / 4, p<0.01$ and Syt $1{ }^{-1 / R N A(S y t 7)} 0.013 \pm 0.001, n=24 / 4, p<0.0001 / 0.01 ;$ Kruskal-Wallis test). These results suggest that SYT7 compensates for the loss of SYT1 dependent synaptic vesicle priming and clamp of spontaneous release (Figure 4A and B). Next, we examined whether SYT7 expression contributes evoked NT release and the probability of vesicle fusion of Syt $1^{-1}$ neurons. While knocking-down of SYT7 expression levels in Syt1 ${ }^{-1}$ neurons had an impact on the amplitude and charge of the $\mathrm{Ca}^{2+}$ evoked release at both stages, the changes were proportional to the observed for the RRP size, and thus did not lead to a further significant effect in the Pvr (Figure 4C and D; (EPSC charge, $p C$ ):

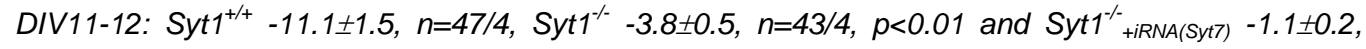
$n=40 / 4, p<0.0001 / 0.001 ;$ DIV15-16: Syt $1^{+/+}-36 \pm 5, n=40 / 4$, Syt1 $1^{-1}-5.3 \pm 1, n=44 / 4, p<0.0001$ and Syt ${ }^{1 / 1}$ +iRNA(Syt7) $-1.4 \pm 0.3, n=32 / 4, p<0.0001 / 0.01$; (Pvr, \%): DIV11-12: Syt1 ${ }^{1++} 8.5 \pm 1, n=47 / 4$, Syt1 1 3.2 \pm 0.5 , $n=40 / 4, p<0.0001$ and Syt1 ${ }_{+i R N A(S y t 7)} 3.3 \pm 0.6, n=39 / 4, \quad p<0.0001 / n s ;$ DIV15-16: Syt1 ${ }^{+/ 4} 7.4 \pm 0.8$,

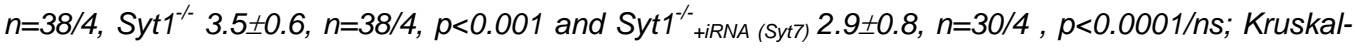
Wallis test) of Syt1 ${ }^{-/}$autaptic neurons in any stages tested.

All together these results suggest that while SYT1 is essential for evoked neurotransmitter release, SYT7 is well capable of substituting in the SYT1's role in clamping spontaneous release and enabling SV priming, which results in weakening of SYT1 deficiency of these two phenotypes in less mature neurons. 
326 Our results on SV priming and spontaneous clamping suggest that the underlying phenotypes are dependent on both SYT1 and SYT7 protein expression levels. Since monoallelic mutations in SYT1 lead to neurological disorders (Baker et al., 2015, 2018), it raises the question of whether and which of the three SYT1 functions in NT release is impaired by reduced SYT1 protein amount. We first

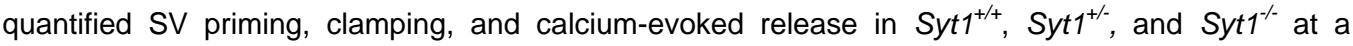
maturation stage where SYT1 dominates the synaptic phenotype (DIV15-21).

Immunocytochemistry experiments showed that the amount of SYT1 protein at presynaptic terminals of Syt1 $1^{+/}$neurons was $\sim 50 \%$ reduced compared with Syt $1^{+/+}$neurons (Figure 5A and B;

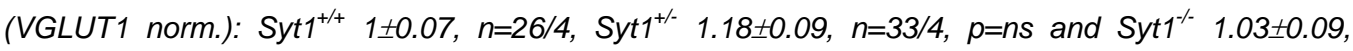
$n=31 / 4, p=n s / n s ;\left(S Y T 1 /\right.$ VGLUT1 norm.): Syt $1^{++} 1 \pm 0.06, n=26 / 4$, Syt $1^{+/} 0.62 \pm 0.04, n=33 / 4, p<0.01$ and Syt1 $1^{-1} 0.18 \pm 0.02, n=31 / 4, p<0.0001 /<0.0001 ;$ Kruskal-Wallis test). As expected, the complete absence of SYT1 in Syt1 ${ }^{-/}$neurons resulted in a $\sim 40 \%$ reduction in the pool size (Figure 5C). However, we found no significant differences in the RRP size between Syt1 ${ }^{+/+}$and Syt1 $1^{+/}$neurons (Figure 5C; $(R R P, p C)$ : Syt1 ${ }^{+/+} 330 \pm 24, n=92 / 4$, Syt1 ${ }^{+/} 382 \pm 26, n=87 / 4, p=n s$ and Syt1 $180 \pm 15$, $n=91 / 4, p<0.0001 /<0.0001$; Kruskal-Wallis test). This indicates that loss of $>50 \%$ of SYT1 protein is required to impair SYT1's role in SV priming. A significant increase in the spontaneous release rate of synaptic vesicles was observed in Syt $1^{+/}$autaptic neurons (Figure 5D; (Spontaneous rate, $s^{-1}$ ) Syt $1^{+/+}$ $0.002 \pm 0.0002, n=61 / 4$, Syt1 ${ }^{+/} 0.004 \pm 0.0004, n=68 / 4, p<0.05$ and Syt1 $1^{-/} 0.007 \pm 0.0007, n=77 / 4$, $p<0.0001 /<0.0001 ;$ Kruskal-Wallis test).

In agreement with previous work (Geppert et al., 1994; Nishiki and Augustine, 2004; Xue et 346 al., 2008), autaptic Syt $1^{-}$neurons showed severe desynchronization of evoked release, which is 347 reflected in the drastic reduction in peak EPSC amplitude (Figure 5E). Furthermore, integrating the 348 charge of the EPSC over 1s past AP triggering, we also observed a $75 \%$ decrease in the EPSC charge (Figure 5F). In contrast, we detected no significant difference in Syt1 $1^{+/}$EPSC amplitude and 350 charge when compared with $S y t 1^{+/+}$autaptic neurons (Figure 5E and F; (EPSC amplitude, nA):

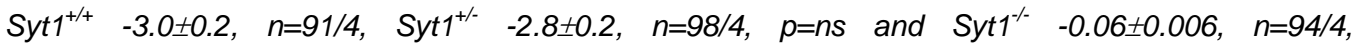
$p<0.0001 /<0.0001$; Kruskal-Wallis test; (EPSC charge, $p C$ ): Syt1 ${ }^{+/+}-24 \pm 2, n=91 / 4$, Syt1 ${ }^{+/}-23 \pm 2$, $n=98 / 4, p=n s$ and Syt1 ${ }^{-/}-5 \pm 0.5, n=94 / 4, p<0.0001 /<0.0001$; Kruskal-Wallis test). We then examined 354 whether $50 \%$ loss of SYT1 protein leads to some changes in EPSC kinetics in the Syt1 $1^{+/}$neurons. 
The EPSC rise time of $S y t 1^{+/}$neurons was like wild-type, while the rise time was $\sim 3$ fold slower for Syt1 $1^{-/}$neurons (Figure 5G; (EPSC rise time, ms): Syt $1^{+/+} 0.9 \pm 0.04, n=83 / 4, S y t 1^{+/} 0.9 \pm 0.04, n=83 / 4$, $p=n s$ and Syt $1^{-/} 2.8 \pm 0.3, n=65 / 4, p<0.0001 /<0.0001 ;$ Kruskal-Wallis test). The decay time of EPSC was analyzed by a two-exponential fit, and we found that between the Syt $1^{+/+}$and $S y t 1^{+/-}$neurons, both the fast and slow component was unaltered in their relative amplitudes and time constants (Figure $\mathbf{5 H}$ ). In contrast, in Syt1 $1^{-/}$neurons time constant increased, and the fractional contribution of the slow component increased from $20 \%$ to $80 \%$ (Figure $5 \mathbf{H}$; (EPSC components): Syt ${ }^{+/+}$(slow) $0.15 \pm 0.02$ (fast) $0.85 \pm 0.02, n=71 / 4, S y t 1^{+-}$(slow) $0.18 \pm 0.01$ (fast) $0.82 \pm 0.01, n=68 / 4, p=0.1705$ and Syt1 ${ }^{-/}$(slow) $0.85 \pm 0.05$ (fast) $0.15 \pm 0.05, n=78 / 4, p<0.0001 /<0.0001 ;$ Kruskal-Wallis test).

The reduction of EPSC charge in Syt1 $1^{-}$exceeds the reduction in RRP size, indicating that complete loss of SYT1 leads to a reduction in vesicular release probability. We thus measured vesicular release probability in Syt1 ${ }^{+/}$neurons and surprisingly found a significant reduction in Pvr

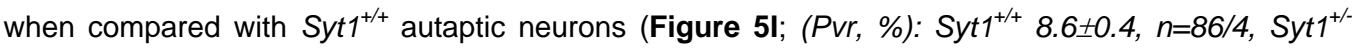
7.5 $\pm 0.6, n=86 / 4, p<0.05$ and Syt1 $1^{-/} 4.4 \pm 0.5, n=90 / 4, p<0.0001 /<0.0001 ;$ Kruskal-Wallis test). The reduced release probability was corroborated by analysis of short-term plasticity experiments, in which we applied sequential pairs of action potentials with an interpulse interval of 25,50 , or 100 ms. Consistent with the Pvr results, Syt1 $1^{-}$neurons displayed a significant increase of the paired-pulsed ratio (PPR) at 10, 20 and $40 \mathrm{~Hz}$ compared with $S y t 1^{+/+}$and a significant increase of the PPR at 20 and

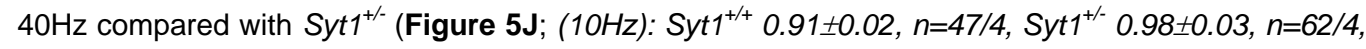
$p=n s$ and Syt1 $1.38 \pm 0.09, n=41 / 4, p<0.0001 /<0.0001 ;$ Kruskal-Wallis test; $(20 \mathrm{~Hz}):$ Syt $1^{+/+} 0.90 \pm 0.02$, $n=53 / 4$, Syt $1^{+/} 0.99 \pm 0.02, n=50 / 4, p<0.05$ and Syt1 $1^{-/} 1.51 \pm 0.13, n=20 / 4, p<0.0001 /<0.0001 ;$ Kruskal-

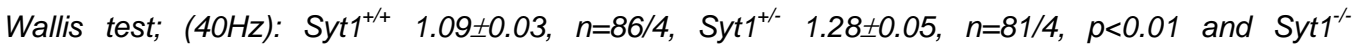
3.46 $\pm 0.37, n=78 / 4, p<0.0001 /<0.0001$; Kruskal-Wallis test). In addition, we performed $20 \mathrm{AP}$ at $20 \mathrm{~Hz}$ train-pulses experiment. Syt1 $1^{-/}$neurons strongly facilitated, and even Syt1 $1^{+/}$neurons showed significantly less depression compared to $S y t 1^{+/+}$neurons (Figure $5 \mathrm{~K}$; Syt $1^{+/+} 0.55 \pm 0.03, n=53 / 4$, Syt $1^{+/} 0.70 \pm 0.04, n=50 / 4, p<0.01$ and Syt1 ${ }^{-/} 4.7 \pm 0.6, n=20 / 4, p<0.0001 ;$ Kruskal-Wallis test).

Together, these results demonstrate that the $50 \%$ reduced SYT1 protein at Syt $1^{+/-}$presynaptic terminals did not affect the RRP size, but modestly impaired release efficiency and the rate of spontaneous release. Although isn't clear whether the patients carrying a heterozygous mutation in 
the SYT1 gene may have an altered SYT1 presence at the glutamatergic synapses, these findings may be relevant to understand how a $50 \%$ deficiency in SYT1 expression could contribute to develop an aberrant neurological phenotype.

\section{Synaptotagmin-1 is a limiting factor for vesicle fusion release efficiency during high-frequency} stimulation

Our analysis with the Syt1 ${ }^{+/}$neurons showed that $50 \%$ reduced STY1 expression affects synaptic properties such as spontaneous release rate and efficiency of the release, indicating the relative sensitivity of SYT1 protein levels on release efficacy. We extended this analysis to overexpression of the SYT1. We used a dose of lentivirus to mediate SYT1 overexpression in Syt1 ${ }^{+/+}$autaptic neurons (DIV15-21), which led to an overexpression of $~ 75 \%$ of WT levels in SYT1 content in presynaptic terminals (Figure 6A; (VGLUT1 norm.): Syt1 ${ }^{+/+} 1 \pm 0.04, n=30 / 3$ and Syt $1^{+/+}+$SYT1 1.06 $\pm 0.04, n=30 / 3$, $p=0.29$; (SYT1/VGLUT1 norm.): Syt1 ${ }^{+/+} 1 \pm 0.05, n=30 / 3$ and Syt1 ${ }^{+/+}{ }_{+S Y T 1} 1.78 \pm 0.06, n=30 / 3, p<0.0001$;

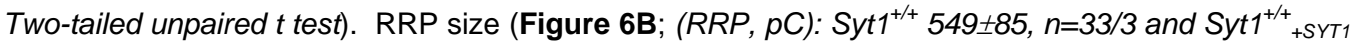
$500 \pm 57, n=36 / 3, p=0.67$; Mann-Whitney test), spontaneous release rate (Figure 6C; (Spontaneous rate, $\left.s^{-1}\right):$ Syt $1^{+/+} 0.003 \pm 0.0005, n=31 / 3$ and Syt $1^{+/+}{ }_{+S Y T 1} 0.0017 \pm 0.0002, n=34 / 3, p=0.45$; MannWhitney test) and the EPSC amplitude (Figure 6D; (EPSC amplitude, $n A$ ): Syt $t 1^{+/+}-2.99 \pm 0.43, n=34 / 3$ and Syt1 $^{+/+}{ }_{+S Y T 1}-4.10 \pm 0.47, n=37 / 3, p=0.084$; Mann-Whitney test) were statistically not significant compared to Syt1 ${ }^{+/+}$neurons (DIV15-21). Also computing Pvr in the SYT1 overexpressing neurons did not show any significant change (Figure 6E; (Pvr, \%): Syt1 ${ }^{+/+} 7.0 \pm 1.1, n=33 / 3$ and Syt $1^{+/+}{ }_{+S Y T 1}$ 8.4 $\pm 1.0, n=35 / 3, \quad p=0.19$; Mann-Whitney test). However, SYT1 overexpression caused more depression of the second response during paired-pulse experiments (Figure 6F; $(4 \mathrm{OHz})$ : Syt1 ${ }^{1 /+}$ $1.26 \pm 0.06, n=34 / 3$ and $S y t 1^{+/ 4}{ }_{+S Y T 1} 0.98 \pm 0.04, n=33 / 3, p=0.0005$; Mann-Whitney test) compared to Syt $1^{++}$, emphasizing that release efficacy is more sensitive to changes in SYT1 levels than its function in regulating $R R P$ size.

\section{Manipulation of endogenous expression levels of Synaptotagmin-1 reveals different functional}

\section{thresholds}

Does SYT1 regulate NT-release and recruitment of SVs to the presynaptic PM in a protein concentration dependent manner? To address this question, we examined the impact of titrating endogenous SYT1 protein expression in autaptic neurons at DIV15-21 using RNA interference (RNAi) 
413 technology, which led to a graded reduction of SYT1 levels in presynaptic terminals down to $10-25 \%$

414 of $S y t 1^{+/+}$SYT1 expression levels (Figure 7A; (VGLUT1 norm.): Syt1 $1^{+++} 1 \pm 0.06, \quad n=19 / 3$,

$415 S y t 1^{+/+}{ }_{1 \times R N A(S y t 1)} 1.32 \pm 0.1, n=20 / 3, p=n s, S_{1} y t 1^{+/+}{ }_{2 x R N A(S y t 1)} 1.26 \pm 0.09, n=24 / 3, p=n s$ and $S y t 1^{+/+}{ }_{4 \times R N A(S y t 1)}$

416 1.33 $\pm 0.10, n=27 / 3, p=n s ;\left(S Y T 1 /\right.$ VGLUT1 norm.): Syt $1^{+/+} 1 \pm 0.05, n=19 / 3$, Syt $1^{+/+}{ }_{1 \times R N A(S y t 1)} 0.25 \pm 0.04$,

$417 n=20 / 3, \quad p<0.001, \quad S y t 1^{+/+}{ }_{2 \times R N A(S y t 1)} 0.13 \pm 0.02, n=24 / 3, \quad p<0.0001$ and Syt $1_{4 \times R N A(S y t 1)}^{+/+} 0.096 \pm 0.01$, $418 \quad n=27 / 3, p<0.0001 ;$ Kruskal-Wallis test).

We found that the number of fusion-competent vesicles, as measured by sucrose application, was only significantly reduced when the SYT1 protein expression level was below 10\% (Figure 7B; (RRP norm.): Syt $1^{+/+} 1 \pm 0.11, n=51 / 4, S y t 1^{+/+}{ }_{1 \times R N A(S y t 1)} 0.97 \pm 0.13, n=35 / 4, p=n s, \quad S y t 1^{+/+}{ }_{2 x R N A(S y t 1)}$ $0.83 \pm 0.11, n=37 / 4, p=n s$ and $S_{t} 1^{+/+}{ }_{4 \times R N A(S y t 1)} 0.61 \pm 0.08, n=39 / 4, p<0.05 ;$ Kruskal-Wallis test). On the other hand, spontaneous release rate was affected by the gradual reduction in SYT1 expression levels, showing progressive unclamping with reduction of SYT1 expression, reaching a 3-fold increase when SYT1 levels were close to those of Syt1 ${ }^{-1}$ (Figure 7C; (Spontaneous rate norm.): $S_{S y t 1^{+/+}} 1 \pm 0.11, n=55 / 4, \quad S y t 1^{+/+}{ }_{1 \times R N A(S y t 1)} 1.54 \pm 0.16, n=40 / 4, \quad p<0.05, \quad S y t 1^{+/+}{ }_{2 \times R N A(S y t 1)} 1.67 \pm 0.23$, $n=35 / 4, \quad p<0.05$ and $\left.S_{t} 1^{+/+}{ }_{4 \times R N A(S y t 1)} 2.4 \pm 0.47, n=41 / 4, p<0.0001 ; K r u s k a l-W a l l i s ~ t e s t\right)$. Processes 428 involved in evoked release showed an even higher sensitivity to SYT1 expression levels (Figure 7D429 G). The EPSC charge as well as the fast synchronous component of release were significantly 430 reduced with all levels of SYT1 knock-down tested (Figure 7D and E; (EPSC charge norm.): Syt1 ${ }^{+/+}$ $1 \pm 0.11, n=57 / 4, \quad S y t 1^{+/+}{ }_{1 \times R N A(S y t 1)} 0.58 \pm 0.1, \quad n=36 / 4, \quad p<0.01, \quad$ Syt $1^{+/+}{ }_{2 \times R N A(S y t 1)} 0.36 \pm 0.06, \quad n=39 / 4$, $p<0.0001$ and Syt $1_{4 \times R N A(S y t 1)}^{+/+} 0.32 \pm 0.05, n=43 / 4, p<0.0001 ;$ Kruskal-Wallis test; (EPSC components): Syt1 ${ }^{+/+}$(slow) $0.28 \pm 0.02$ (fast) $0.72 \pm 0.02, n=56 / 4$, Syt $1^{+/+}{ }_{1 \times R N A(S y t 1)}$ (slow) $0.61 \pm 0.06$ (fast) $0.39 \pm 0.06$, $n=29 / 4, \quad p<0.0001, \quad S y t 1^{+/+}$2xRNA(Syt1) (slow) $0.66 \pm 0.04$ (fast) $0.34 \pm 0.04, \quad n=39 / 4, \quad p<0.0001$ and Syt $^{+/+}{ }_{4 \times R N A(S y t 1)}$ (slow) $0.65 \pm 0.04$ (fast) $0.35 \pm 0.04, n=40 / 4, p<0.0001$; One-way ANOVA test). 436 Additionally, the release efficacy was significantly reduced in all knocked-down groups, demonstrated 437 by a reduced Pvr and increased paired-pulse ratio compared with Syt1 ${ }^{+/+}$(Figure 7F and G; (Pvr 438 norm.): $S_{t} t 1^{+/+} 1 \pm 0.08, n=51 / 4, S_{t} 1^{+/+}{ }_{1 x R N A(S y t 1)} 0.73 \pm 0.12, n=30 / 4, p<0.01, S y t 1^{+/+}{ }_{2 x R N A(S y t 1)} 0.52 \pm 0.07$, $n=31 / 4, \quad p<0.0001$ and Syt $1_{4 x R N A(S y t 1)}^{+/+} 0.5 \pm 0.07, n=39 / 4, \quad p<0.0001 ; \quad\left(P P R 40 H z\right.$, norm.): Syt1 $1^{+/+}$ 440 $1 \pm 0.05, n=51 / 4, S y t 1_{1 \times R N A(S y t 1)}^{+/+} 1.84 \pm 0.18, n=34 / 4, p<0.001, S y t 1_{2 \times R N A(S y t 1)}^{+/+} 2.16 \pm 0.19, n=33 / 4$, $p<0.0001$ and $S y t 1^{+/+}{ }_{4 \times R N A(S y t 1)} 2.67 \pm 0.35, n=39 / 4, p<0.0001 ;$ Kruskal-Wallis test). To exclude that 
442 these results might be due to the number of viral particles used, we manipulated SYT1 endogenous 443 protein expression in Syt1 ${ }^{+/}$neurons (DIV15-21) using the same Syt1 RNAi construct (Figure 8). We 444 used half of the amount of viral infection units to reduce the expression to approximately the same 445 levels as in the titration experiments in the Syt1 $1^{+/+}$neurons (Figure 8A; (VGLUT1 norm.): Syt1 $1^{+-}$

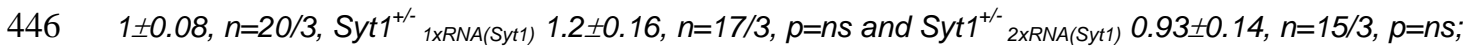

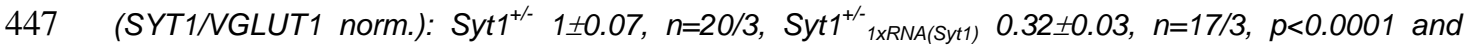
448 Syt1 ${ }^{+/}$2xRNA(Syt1) $0.22 \pm 0.02, n=15 / 3, p<0.0001 ;$ Kruskal-Wallis test). All electrophysiological parameters 449 measured showed the same trend as our experiments performed on Syt $1^{+/+}$neurons (Figure 8B-G; 450 (RRP norm.): Syt $1^{+/+} 1 \pm 0.16, n=21 / 3$, Syt $1^{+/+}{ }_{1 \times R N A(S y t 1)} 0.72 \pm 0.14, n=22 / 3, p=n s$ and Syt $1^{+/+}{ }_{2 \times R N A(S y t 1)}$ 451

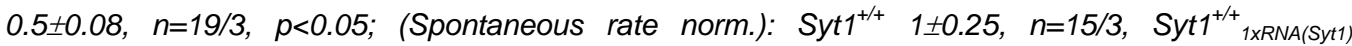
$1.70 \pm 0.39, n=18 / 3, p=n s$ and $S y t 1^{+/+}{ }_{2 \times R N A(S y t 1)} 3.21 \pm 1.09, n=15 / 4, p<0.05$; (EPSC charge norm.): Syt1 $1^{+/+} 1 \pm 0.15, n=29 / 3, S_{y t 1}{ }^{+/+}{ }_{1 \times R N A(S y t 1)} 0.35 \pm 0.06, n=23 / 3, p<0.001$ and $S y t 1^{+/+}{ }_{2 \times R N A(S y t 1)} 0.29 \pm 0.05$, $n=21 / 4, \quad p<0.0001 ; \quad$ (EPSC components): Syt1 ${ }^{1 /+}$ (slow) $0.27 \pm 0.04$ (fast) $0.73 \pm 0.04, n=21 / 3$, Syt $1^{+/ t}{ }_{1 \times R N A(S y t 1)}$ (slow) $0.62 \pm 0.08$ (fast) $0.38 \pm 0.08, n=18 / 3, p<0.01$ and $S y t 1^{+/+}{ }_{2 \times R N A(S y t 1)}$ (slow) $0.64 \pm 0.07$ (fast) $0.36 \pm 0.07, n=20 / 3, p<0.001$; (Pvr norm.): Syt ${ }^{1++} 1 \pm 0.12, n=22 / 3$, Syt $1^{+/+}{ }_{1 \times R N A(S y t 1)}$ $0.62 \pm 0.11, n=21 / 3, p<0.05$ and $S y t 1^{+/ t}$ 2xRNA(Syt1) $0.57 \pm 0.09, n=20 / 3, p<0.01$; (PPR4OHz, norm.): Syt $1^{1 /+}$ $1 \pm 0.09, n=27 / 3$, Syt ${ }^{+/+}{ }_{1 \times R N A(S y t 1)} 2.47 \pm 0.28, n=22 / 3, p<0.0001$ and $S y t 1^{+/+}{ }_{2 \times R N A(S y t 1)} 2.41 \pm 0.2, n=17 / 3$, $p<0.0001 ;$ Kruskal-Wallis test).

Our previous results suggest different relationships between synaptic functions and SYT1 expression levels. To understand these relationships in greater detail, we used our wide range of SYT1 protein levels measurements at the presynaptic terminals of glutamatergic neurons (DIV15-21) to construct dose-response curves for different release properties: RRP size, spontaneous release rate, EPSC charge and Pvr (Figure 7 H-K) and fitted our experimental data with a standard Hill equation (see Materials and Methods). To illustrate how SYT7 may potentially modulate SYT1's synaptic functions and to illustrate putative redundant function between SYT1 and SYT7, we included the normalized data from the SYT7 knock-down experiments performed on Syt ${ }^{-1 /}$ neurons from Figure 4 (DIV15-16). However, the SYT7 data point was excluded from the curve fitting of the SYT1 expression-function relation.

First, the function of SYT1 in synaptic vesicle priming was least sensitive to protein loss 471 (Figure $7 \mathrm{H}$ ), since even an $85 \%$ decrease in SYT1 protein expression at the synapse still displayed 
472 normal priming function. The best-fit value $\mathrm{Kd}$ for this parameter of 0.02 demonstrates that only when

473 SYT1 is almost absent from the synapse, RRP size decreases (Figure 7H). SYT1's function as a

474 regulator of spontaneous NT-release (Figure 7I) showed a higher sensitivity to protein levels, leading

475 to increased spontaneous release activity when the expression levels were reduced by $50 \%$ or more.

476 Evoked release was the most sensitive to the variations of SYT1 expression, as reflected both by 477 assessing EPSC charge and Pvr (Figure 7J and K). The similarity of the EPSC charge and Pvr 478 functions is not surprising since both reflect SYT1's role as calcium sensor for evoked release. The $479 \mathrm{Kd}$ value $(0.47)$ for the EPSC charge fit indicates that genetic modification of SYT1 expression 480 associated with allelic loss or gene duplication may lead to more pronounced impact on evoked neurotransmitter release compared to SYT1 functions in vesicle priming or suppression of 482 stoichiometries.

\section{Discussion}

In this study, we iterated the existence of three different synaptic functions performed by SYT1 using an autaptic primary neuronal culture model. Previous results concerning the ability of SYT1 to promote SV docking/priming (Geppert et al., 1994; Jorgensen et al., 1995; Reist et al., 1998; Nagy et al., 2006; Liu et al., 2009; Bacaj et al., 2013, 2015; Imig et al., 2014; Chang et al., 2018; Huson et al., 2020) and spontaneous release clamping (DiAntonio and Schwarz, 1994; Geppert et al., 1994;

491 Littleton et al., 1994; Mackler et al., 2002; Yoshihara and Littleton, 2002; Chicka et al., 2008; Liu et al., 492 2009; Xu et al., 2009; Bacaj et al., 2013; Wierda and Sørensen, 2014) have been contradictory. Here, 493 we demonstrated that SYT1 has a role in SV priming and clamping spontaneous release, which becomes essential over neuronal maturation. More mature SYT1-lacking hippocampal glutamatergic neurons showed a deficit in the RRP size and an increase in the spontaneous release rate, while early autaptic cultures did not (Table 1). Therefore, the time point and neuronal maturation stage at which experiments were performed could explain some discrepancies in previous work. What factor could compensate for SYT1 loss at early stages? We found that SYT7 has partially overlapping

500 and we conclude that at an early neuronal stage endogenous SYT7 protein could compensate for the 501 loss of SYT1 in calcium-independent SV functions, such as priming and clamping of spontaneous 
release. When both proteins were missing at the presynaptic terminal, regardless of the culture stage,

503 the pool of SVs was consistently reduced and spontaneous release was significantly increased.

504 To dissect the specific interaction of the different mechanisms of NT-release and to better

505 understand SYT1's functions beyond that in synchronous release, we investigated the impact of 506 systematically varying SYT1 expression levels on SV priming, clamping, and evoked release. SV priming was impaired only after a major drop in SYT1 concentration, suggesting that this process, while ultimately affected by the presence of SYT1, was relatively insensitive to changes in the number of SYT1 molecules. Varying SYT1 protein concentration also had a rather moderate impact on spontaneous release compared to the graded impact of varying SYT1 concentration on calcium triggered release processes. Accordingly, heterozygotic Syt1 $1^{+/}$excitatory hippocampal neurons with a $50 \%$ reduction in SYT1 protein expression in the presynaptic terminal showed no effect on RRP size compared to WT SYT1 protein levels, while calcium-dependent release efficacy was impaired by the $50 \%$ SYT1 protein reduction in Syt $1^{+/}$neurons. Hence, when we applied a dose-response model to illustrate the sensitivity of the priming function to SYT1 expression the curve described has a hyperbolic-like shape, where small amounts of SYT1 protein expression are sufficient to preserve maximum priming activity. Our overall analysis of the sensitivity of synaptic processes to SYT1 protein levels renders a rank where the least sensitive function is SV priming, followed by clamping of spontaneous and, finally, calcium-evoked release. Do the differences in sensitivity to SYT1 protein levels of these three synaptic processes indicate that SYT1 is involved in three consecutive and independent pathways? Due to the clear difference in sensitivity to expression levels for priming compare to evoked release we think that SYT1 is affecting at least two pathways, in addition to its possible roles in endocytosis (Poskanzer et al., 2003; Yao et al., 2012; Liang et al., 2017). One hypothesis is that SYT1 undergoes different conformational stages to regulate different synaptic processes. Indeed, it has been suggested that SYT1 C2B domain-dependent oligomerization provides the molecular basis for SYT1 control of spontaneous and asynchronous release, and upon influx of calcium SYT1 oligomers undergo a conformational change from an SV clamping mode to a mode which allows the triggering of synchronous release (Bello et al., 2018; Tagliatti et al., 2020). SYT1 protein level titration experiments not only revealed to us the distinct roles of SYT1, but may also could contribute to the understanding of the pathophysiological mechanisms underlying SYT1-associated neurodevelopmental disorders (Baker et al., 2015, 2018; Bradberry et al., 2020). In 
532 our study, we show that haploinsufficiency of SYT1 resulted in an aberrant spontaneous release 533 phenotype and decreased release probability with additional consequences in short-term plasticity 534 characteristics. If patients with heterozygous SYT1 mutations that has been described to produce a 535 SYT1 loss-of-function (Bradberry et al., 2020), should occur a reduced SYT1 protein level, this could 536 lead to an enhance spontaneous release or a decreased release probability and, ultimately, contribute 537 to network disfunction. It has been reported that at least one of the de novo SYT1 mutations $538\left(S Y T 1_{\text {Mзозк }}\right)$ is expressed at lower level than endogenous wild-type protein and failed to localize at 539 nerve terminals (Baker et al., 2018). Furthermore, two of the variants (SYT1 D304G $_{\text {and }}$ SYT1 D366E $_{\text {) }}$ 540 failed to efficiently relocalize to nerve terminals following stimulation (Baker et al., 2018). While allelic 541 expressivity may not explain the whole pathophysiology of the SYT1-associated neurodevelopmental 542 disorder it could exacerbate synaptic manifestations of individual SYT1 variants (Baker et al., 2018; 543 Bradberry et al., 2020). In fact, a recent article revealed aberrant spontaneous NT release with some 544 mutations from SNAP25-associated encephalopathies, indicating that when this form of release is 545 affected it could result in developmental and epileptic encephalopathies (Alten et al., 2021). 546 Conversely, our findings may indicate that the patient's pathophysiology that could derive from 547 changes in the SYT1 expression levels are unlikely to be related to the priming of synaptic vesicles 548 function at the presynaptic terminal.

While SYT1's function as a calcium sensor for NT release has been extensively proven, the role for SYT7 is less clear. SYT7 has been suggested as the calcium sensor for asynchronous release (Maximov et al., 2008; Bacaj et al., 2013; Luo et al., 2015; Turecek and Regehr, 2018). SYT7

552 has also been proposed as a mediator of short-term facilitation of transmission during repetitive 553 stimulation (Wen et al., 2010; Jackman et al., 2016; Chen et al., 2017b; Fujii et al., 2021), involving a 554 mechanism of SYT7's concerted action with SYT1 on the fusion energy barrier (Schotten et al., 2015; Chen et al., 2017b; Jackman and Regehr, 2017; Huson et al., 2020). Supporting its role as a calcium sensor, SYT7 contributes to regulated exocytosis in chromaffin and pancreatic cells (Sugita et al., 2001; Shin et al., 2002; Schonn et al., 2008; Bendahmane et al., 2020). Additionally, it has been reported that SYT7 functions as a $\mathrm{Ca}^{2+}$-sensor for synaptic vesicle replenishment (Liu et al., 2014; Silva et al., 2021). In our study, we found no direct evidence that SYT7 is involved in regulating calcium-dependent release. However, we showed that suppressing SYT7 expression on Syt $1^{-1-}$ neurons further decreased evoked neurotransmitter release, and the analysis of RRP size reveals 
that the reduction in evoked response is rather due to the priming action of SYT7, and not due to an effect on calcium triggered release. It is quite possible that putative competition between SYT1 and SYT7 may contribute to the observed modulation of calcium triggered NT release.

This study could be framed within a series of works that aim to understand how synapses respond to the relative changes in the expression of different presynaptic components and to study the consequences on neurotransmission (Arancillo et al., 2013; Zarebidaki et al., 2020). Although mammalian and invertebrate loss-of-function mutants of presynaptic proteins continues to provide deep insights of their role in neurotransmitter release (Geppert et al., 1994; Schulze et al., 1995; Deng et al., 2011), a more sophisticated model where there is a control of protein amount has been proven to be a powerful approach. For instance, examining the Munc13-1 concentration dependency of priming showed that RIM boosts Munc13-1's priming function (Zarebidaki et al., 2020). Another example comes from the study of Syntaxin-1 (Stx1), where to titrate down Stx1's protein expression levels revealed that priming and vesicle fusion are likely governed by highly related mechanisms (Arancillo et al., 2013). Here, therefore we advocate for this experimental approach as a useful way to obtain a quantitative understanding of how the different elements of the presynaptic release machinery differently regulate the steps involved in the process of synaptic transmission.

Overall, to precisely understand the distinct functions of SYT1, variations in the expression and balance between SYT1 and SYT7 at SVs and the PM over neuronal stages should be taken into consideration. Also, our data demonstrated that both SYT1 and SYT7 are capable of SV priming and clamping. Likely the efficient execution of these functions relies on their intermolecular interactions with both SNAREs and phospholipids from the PM (Perin et al., 1990; Chapman et al., 1995; Li et al., 1995; Sugita et al., 2002; Zhou et al., 2017). Further study of how the interplay between these proteins affects the intrinsic speed of neurotransmitter release is needed. Moreover, we emphasize that when studying disorders associated with presynaptic protein mutations, all these parameters could be conditioning the quantitative aspects of the cellular phenotype and, in turn, the pathophysiology that derives from it.

\section{References}

Alten B, Zhou Q, Shin OH, Esquivies L, Lin PY, White KI, Sun R, Chung WK, Monteggia LM, Brunger AT, Kavalali ET (2021) Role of Aberrant Spontaneous Neurotransmission in SNAP25-Associated Encephalopathies. Neuron 109(1):59-72.e5. 
Arancillo M, Min S, Gerber S, Mu A, Wu Y, Herman M, Trimbuch T, Rah J, Ahnert-hilger G, Riedel D, Su TC (2013) Titration of Syntaxin1 in Mammalian Synapses Reveals Multiple Roles in Vesicle Docking, Priming, and Release Probability. J Neurosci 33:16698-16714.

Bacaj T, Wu D, Burré J, Malenka RC, Liu X, Südhof TC (2015) Synaptotagmin-1 and -7 Are Redundantly Essential for Maintaining the Capacity of the Readily-Releasable Pool of Synaptic Vesicles. PLoS Biol 13(10):e1002267.

Bacaj T, Wu D, Yang X, Morishita W, Zhou P, Xu W, Malenka RC, Südhof TC (2013) Asynchronous Phases of Neurotransmitter Release. Neuron 80:947-959.

Baker K, Gordon SL, Melland H, Bumbak F, Scott DJ, Jiang TJ, Owen D, Turner BJ, Boyd SG, Rossi M, AIRaqad M, Elpeleg O, Peck D, Mancini GMS, Wilke M, Zollino M, Marangi G, Weigand H, Borggraefe I, Haack T, Stark Z, Sadedin S; Broad Center for Mendelian Genomics, Tan TY, Jiang Y, Gibbs RA, Ellingwood S, Amaral M, Kelley W, Kurian MA, Cousin MA, Raymond FL (2018) SYT1-associated neurodevelopmental disorder: A case series. Brain 141:2576-2591.

Baker K, Gordon SL, Grozeva D, Van Kogelenberg M, Roberts NY, Pike M, Blair E, Hurles ME, Chong WK, Baldeweg T, Kurian MA, Boyd SG, Cousin MA, Raymond FL (2015) Identification of a human synaptotagmin-1 mutation that perturbs synaptic vesicle cycling. J Clin Invest 125:1670-1678.

Bekkers JM, Stevens CF (1991) Excitatory and inhibitory autaptic currents in isolated hippocampal neurons maintained in cell culture. Proc Natl Acad Sci U S A 88:7834-7838.

Bello OD, Jouannot O, Chaudhuri A, Stroeva E, Coleman J, Volynski KE, Rothman JE, Krishnakumar SS (2018) Synaptotagmin oligomerization is essential for calcium control of regulated exocytosis. Proc Natl Acad Sci U S A 115:E7624-E7631.

Bendahmane M, Morales A, Kreutzberger AJB, Schenk NA, Mohan R, Bakshi S, Philippe JM, Zhang S, Kiessling V, Tamm LK, Giovannucci DR, Jenkins PM, Anantharam A (2020) Synaptotagmin-7 enhances calciumsensing of chromaffin cell granules and slows discharge of granule cargos. J Neurochem 154:598-617.

Bradberry MM, Courtney NA, Dominguez MJ, Lofquist SM, Knox AT, Sutton RB, Chapman ER (2020) Molecular Basis for Synaptotagmin-1-Associated Neurodevelopmental Disorder. Neuron 107:52-64.

Broadie K, Bellen HJ, DiAntonio A, Littleton JT, Schwarz TL (1994) Absence of synaptotagmin disrupts excitation-secretion coupling during synaptic transmission. Proc Natl Acad Sci U S A 91:10727-10731.

Brose N, Petrenko AG, Sudhof TC, Jahn R (1992) Synaptotagmin: A calcium sensor on the synaptic vesicle surface. Science 256:1021-1025.

Chang S, Trimbuch T, Rosenmund C (2018) Synaptotagmin-1 drives synchronous Ca 2+ -triggered fusion by C 2 B-domain-mediated synaptic-vesicle-membrane attachment. Nat Neurosci 21:33-42.

Chapman ER, Davis AF (1998) Direct interaction of a Ca2+-binding loop of synaptotagmin with lipid bilayers. J Biol Chem 273:13995-14001. 
Chapman ER, Hanson PI, An S, Jahn R (1995) Ca2+ regulates the interaction between synaptotagmin and syntaxin 1. J Biol Chem 270:23667-23671.

Chen C, Arai I, Satterfield R, Young SM, Jonas P (2017a) Synaptotagmin 2 Is the Fast Ca2+ Sensor at a Central Inhibitory Synapse. Cell Rep 18:723-736.

Chen C, Jonas P (2017) Synaptotagmins: That's Why So Many. Neuron 94:694-696.

Chen C, Satterfield R, Young SM, Jonas P (2017b) Triple Function of Synaptotagmin 7 Ensures Efficiency of High-Frequency Transmission at Central GABAergic Synapses. Cell Rep 21:2082-2089.

Chicka MC, Hui E, Liu H, Chapman ER (2008) Synaptotagmin arrests the SNARE complex before triggering fast, efficient membrane fusion in response to Ca2+. Nat Struct Mol Biol 15:827-835.

de Wit H, Walter AM, Milosevic I, Gulyás-Kovács A, Riedel D, Sørensen JB, Verhage M (2009) Synaptotagmin-1 Docks Secretory Vesicles to Syntaxin-1/SNAP-25 Acceptor Complexes. Cell 138:935-946.

Deng L, Kaeser PS, Xu W, Südhof TC (2011) RIM proteins activate vesicle priming by reversing autoinhibitory homodimerization of munc13. Neuron 69:317-331.

DiAntonio A, Schwarz TL (1994) The effect on synaptic physiology of synaptotagmin mutations in drosophila. Neuron 12:909-920.

Fernández-Chacón R, Königstorfer A, Gerber SH, García J, Matos MF, Stevens CF, Brose N, Rizo J, Rosenmund C, Südhof TC (2001) Synaptotagmin I functions as a calcium regulator of release probability. Nature 410:41-49.

Fernandez I, Araç D, Ubach J, Gerber SH, Shin OH, Gao Y, Anderson RGW, Südhof TC, Rizo J (2001) Threedimensional structure of the synaptotagmin 1 C2B-domain: Synaptotagmin 1 as a phospholipid binding machine. Neuron 32:1057-1069.

Fujii T, Sakurai A, Littleton JT, Yoshihara M (2021) Synaptotagmin 7 switches short-term synaptic plasticity from depression to facilitation by suppressing synaptic transmission. Sci Rep 11:1-9.

Geppert M, Archer BT, Sudhof TC (1991) Synaptotagmin II: A novel differentially distributed form of synaptotagmin. J Biol Chem 266:13548-13552.

Geppert M, Goda Y, Hammer RE, Li C, Rosahl TW, Stevens CF, Südhof TC (1994) Synaptotagmin I: a major Ca2+ sensor for transmitter release at a central synapse. Cell 79:717-727.

Huson V, Meijer M, Dekker R, Veer M Ter, Ruiter M, van Weering J, Verhage M, Cornelisse LN (2020) Posttetanic potentiation lowers the energy barrier for synaptic vesicle fusion independently of synaptotagmin-1. Elife 9:1-56.

Imig C, Min SW, Krinner S, Arancillo M, Rosenmund C, Südhof TC, Rhee JS, Brose N, Cooper BH (2014) The Morphological and Molecular Nature of Synaptic Vesicle Priming at Presynaptic Active Zones. Neuron $84: 416-431$.

Jackman SL, Regehr WG (2017) The Mechanisms and Functions of Synaptic Facilitation. Neuron 94:447-464. 
Jackman SL, Turecek J, Belinsky JE, Regehr WG (2016) The calcium sensor synaptotagmin 7 is required for synaptic facilitation. Nature 529:88-91

Jorgensen EM, Hartwieg E, Schuske K, Nonet ML, Jin Y, Horvitz HR (1995) Defective recycling of synaptic vesicles in synaptotagmin mutants of caenorhabditis elegans. Nature 378:196-199.

Katz B (1969) The release of neural transmitter substances (Book, 1969). Springfield, III, Thomas.

Kedar GH, Munch AS, van Weering JRT, Malsam J, Scheutzow A, de Wit H, Houy S, Tawfik B, Söllner TH, Sørensen JB, Verhage M (2015) A post-docking role of synaptotagmin 1-C2B domain bottom residues R398/399 in mouse chromaffin cells. J Neurosci 35:14172-14182.

Kochubey O, Babai N, Schneggenburger R (2016) A Synaptotagmin Isoform Switch during the Development of an Identified CNS Synapse. Neuron 90:984-999.

Li C, Ullrich B, Zhang JZ, Anderson RGW, Brose N, Südhof TC (1995) Ca2 +-dependent and -independent activities of neural and non-neural synaptotagmins. Nature 246:170.

Liang K, Wei L, Chen L (2017) Exocytosis, endocytosis, and their coupling in excitable cells. Front Mol Neurosci 10:1-10.

Littleton JT, Stern M, Perin M, Bellen HJ (1994) Calcium dependence of neurotransmitter release and rate of spontaneous vesicle fusions are altered in Drosophila synaptotagmin mutants. Proc Natl Acad Sci U S A 91:10888-10892.

Liu H, Bai H, Hui E, Yang L, Evans CS, Wang Z, Kwon SE, Chapman ER (2014) Synaptotagmin 7 functions as a Ca2+-sensor for synaptic vesicle replenishment. Elife 3:1-18.

Liu H, Dean C, Arthur CP, Dong M, Chapman ER (2009) Autapses and networks of hippocampal neurons exhibit distinct synaptic transmission phenotypes in the absence of synaptotagmin I. J Neurosci 29:7395-7403

Lois C, Hong EJ, Pease S, Brown EJ, Baltimore D (2002) Germline transmission and tissue-specific expression of transgenes delivered by lentiviral vectors. Science 295:868-872.

Luo F, Bacaj T, Südhof TC (2015) Synaptotagmin-7 is essential for Ca2+-triggered delayed asynchronous release but not for Ca2+-dependent vesicle priming in retinal ribbon synapses. J Neurosci 35:1102411033.

Mackler J, Drummond J, Loewen C, Robinson I, Reist N (2002) The C(2)B Ca(2+)-binding motif of synaptotagmin is required for synaptic transmission in vivo. Nature 418:340-344.

Marquèze B, Boudier JA, Mizuta M, Inagaki N, Seino S, Seagar M (1995) Cellular localization of synaptotagmin I, II, and III mRNAs in the central nervous system and pituitary and adrenal glands of the rat. J Neurosci $15: 4906-4917$.

Maximov A, Lao Y, Li H, Chen X, Rizo J, Sørensen JB, Südhof TC (2008) Genetic analysis of synaptotagmin-7 function in synaptic vesicle exocytosis. Proc Natl Acad Sci U S A 105:3986-3991.

Maximov A, Südhof TC (2005) Autonomous function of synaptotagmin 1 in triggering synchronous release 
independent of asynchronous release. Neuron 48:547-554.

Nagy G, Kim JH, Pang ZP, Matti U, Rettig J, Südhof TC, Sørensen JB (2006) Different effects on fast exocytosis induced by synaptotagmin 1 and 2 isoforms and abundance but not by phosphorylation. $\mathrm{J}$ Neurosci 26:632-643.

Nishiki TI, Augustine GJ (2004) Synaptotagmin I synchronizes transmitter release in mouse hippocampal neurons. J Neurosci 24:6127-6132.

Pang ZP, Sun J, Rizo J, Maximov A, Südhof TC (2006) Genetic analysis of synaptotagmin 2 in spontaneous and Ca 2+-triggered neurotransmitter release. EMBO J 25:2039-2050.

Perin MS, Fried VA, Mignery GA, Jahn R, Südhof TC (1990) Phospholipid binding by a synaptic vesicle protein homologous to the regulatory region of protein kinase C. Nature 345:260-263.

Poskanzer KE, Marek KW, Sweeney ST, Davis GW (2003) Synaptotagmin I is necessary for compensatory synaptic vesicle endocytosis in vivo. Nature 426:559-563.

Reist NE, Buchanan J, Li J, DiAntonio A, Buxton EM, Schwarz TL (1998) Morphologically docked synaptic vesicles are reduced in synaptotagmin mutants of Drosophila. J Neurosci 18:7662-7673.

Rizo J, Xu J (2015) The Synaptic Vesicle Release Machinery. Annu Rev Biophys 44:339-367.

Rosenmund C, Stevens CF (1996) Definition of the readily releasable pool of vesicles at hippocampal synapses. Neuron 16:1197-1207.

Sabatini BL, Regehr WG (1999) Timing of synaptic transmission. Annu Rev Physiol 61:521-542.

Schonn JS, Maximov A, Lao Y, Südhof TC, Sørensen JB (2008) Synaptotagmin-1 and -7 are functionally overlapping Ca2+ sensors for exocytosis in adrenal chromaffin cells. Proc Natl Acad Sci U S A 105:39984003.

Schotten S, Meijer M, Walter AM, Huson V, Mamer L, Kalogreades L, Ter Veer M, Ruiter M, Brose N, Rosenmund C, Sørensen JB, Verhage M, Cornelisse LN (2015) Additive effects on the energy barrier for synaptic vesicle fusion cause supralinear effects on the vesicle fusion rate. Elife 2015:1-25.

Schulze KL, Broadie K, Perin MS, Bellen HJ (1995) Genetic and electrophysiological studies of drosophila syntaxin-1A demonstrate its role in nonneuronal secretion and neurotransmission. Cell 80:311-320.

Shin OH, Rizo J, Südhof TC (2002) Synaptotagmin function in dense core vesicle exocytosis studied in cracked pc12 cells. Nat Neurosci 5:649-656.

Silva M, Tran V, Marty A (2021) Calcium-dependent docking of synaptic vesicles. Trends Neurosci. 44(7):579592.

Sugita S, Han W, Butz S, Lao Y, Liu X, Ferna R, Su TC, Hines H, Na B (2001) Synaptotagmin VII as a plasma membrane $\mathrm{Ca}(2+)$ sensor in exocytosis. Neuron 30:459-473.

Sugita S, Shin OH, Han W, Lao Y, Südhof TC (2002) Synaptotagmins form a hierarchy of exocytotic Ca2+ sensors with distinct Ca2+ affinities. EMBO J 21:270-280. 
Sutton RB, Davletov BA, Berghuis AM, Sudhof TC, Sprang SR (1995) Structure of the first C2 domain of synaptotagmin I: A novel Ca2+/phospholipid-binding fold. Cell 80:929-938.

Tagliatti E, Bello OD, Mendonça PRF, Kotzadimitriou D, Nicholson E, Coleman J, Timofeeva Y, Rothman JE, Krishnakumar SS, Volynski KE (2020) Synaptotagmin 1 oligomers clamp and regulate different modes of neurotransmitter release. Proc Natl Acad Sci U S A 117:3819-3827.

Turecek J, Regehr WG (2018) Synaptotagmin 7 mediates both facilitation and asynchronous release at granule cell synapses. J Neurosci 38:3240-3251.

Watanabe S, Trimbuch T, Camacho-Pérez M, Rost BR, Brokowski B, Söhl-Kielczynski B, Felies A, Davis MW, Rosenmund C, Jorgensen EM (2014) Clathrin regenerates synaptic vesicles from endosomes. Nature 515:228-233.

Wen H, Linhoff MW, McGinley MJ, Li GL, Corson GM, Mandel G, Brehm P (2010) Distinct roles for two synaptotagmin isoforms in synchronous and asynchronous transmitter release at zebrafish neuromuscular junction. Proc Natl Acad Sci U S A 107:13906-13911.

Wierda KDB, Sørensen JB (2014) Innervation by a GABAergic neuron depresses spontaneous release in glutamatergic neurons and unveils the clamping phenotype of synaptotagmin-1. J Neurosci 34:2100-2110.

Xu J, Pang ZP, Shin OH, Südhof TC (2009) Synaptotagmin-1 functions as a Ca2+ sensor for spontaneous release. Nat Neurosci 12:759-766.

Xue M, Ma C, Craig TK, Rosenmund C, Rizo J (2008) The Janus-Faced Nature of the C 2 B Domain Is Fundamental for Synaptotagmin-1 Function. Nat Struct Mol Biol 15:1160-1168.

Xue M, Reim K, Chen X, Chao H, Deng H, Rizo J, Brose N, Rosenmund C (2007) Distinct domains of complexin I differentially regulate neurotransmitter release. Nat. Struct Mol Biol. 14:949-958.

Yao J, Kwon SE, Gaffaney JD, Dunning FM, Chapman ER (2012) Uncoupling the roles of synaptotagmin I during endo-and exocytosis of synaptic vesicles. Nat Neurosci 15:243-249.

Yoshihara M, Littleton JT (2002) Synaptotagmin functions as a calcium sensor to synchronize neurotransmitter release. Neuron 36:897-908.

Zarebidaki F, Camacho M, Brockmann MM, Trimbuch T, Herman MA, Rosenmund C (2020) Disentangling the roles of RIM and Munc13 in synaptic vesicle localization and neurotransmission. J Neurosci 40:9372-9385.

Zhou Q, Zhou P, Wang AL, Wu D, Zhao M, Südhof TC, Brunger AT (2017) The primed SNARE - complexin synaptotagmin complex for neuronal exocytosis. Nature 548:420-425.

\section{Figures/Extended data/Table legends}

Figure 1. Electrophysiological characterization of Synaptotagmin-1 knockout hippocampal glutamatergic autaptic neurons at different time points. (A) Representative sucrose-evoked current traces (left) and summary bar graphs (right) of total current charge of Syt1 $1^{+/+}$(black) and Syt1 ${ }^{1 /}$ (red) neurons evoked by $0.5 \mathrm{M}$ sucrose solution for $5 \mathrm{~s}$ at early (DIV11-12), 
761

762

763

764

765

766

767

intermediate (DIV15-16) and late (DIV20-21) culture stages. (B) Example traces of spontaneous release events (right) and summary bar graphs of spontaneous release rate (left) calculated by dividing the mEPSC frequency by the number of primed synaptic vesicles at different neuronal culture stages. (C) Representative EPSC traces (left) and summary bar graphs of EPSC amplitudes (right) evoked by a $2 \mathrm{~ms}$ depolarization in $2 \mathrm{mM}$ extracellular $\mathrm{Ca}^{2+}$ from autaptic neurons at the three neuronal stages from Syt $1^{+/+}$(black) and $S y t 1^{-/}$(red). Action potentials were blanked for better EPSC illustration and substituted by arrows. (D) Plot of vesicular release probability (Pvr) in \% calculated by dividing the evoked EPSC charge by the sucrose charge at the different neuronal stages. Data are mean + /- SEM. Statistical significance and $p$ values were calculated using the Mann-Whitney $U$ test $\left({ }^{* * *} \mathrm{P} \leq 0.001,{ }^{* * *} \mathrm{P} \leq 0.0001, \mathrm{~ns}=\right.$ not significant).

Figure 2. Evaluation of the synaptic SYT1 functions in Syt1 $1^{-/}$neurons rescued with SYT1. All immunocytochemistry and electrophysiological experiments were done from Syt1 $1^{+/+}$and Syt1\% hippocampal glutamatergic autaptic neurons at DIV15-21. (A) Representative images of hippocampal glutamatergic autaptic neurons of Syt1\% infected with a lentivirus containing a nuclear GFP vector (-) or Syt1. (B) Summary bar graph showing SYT1/VGLUT1 fluorescence normalized to Syt1 ${ }^{+/+}$(right). Summary bar plot of VGLUT1 fluorescence (left) and normalized summary bar plot of SYT1/VGLUT1 fluorescence intensity. (C) Bar plot of RRP size estimated by application of a hypertonic solution of Syt1 $1^{-\%}$ neurons rescued with SYT1 normalized to the Syt $1^{+/+}$control. (D) Summary bar plot of spontaneous release rate from Syt1\% neurons rescued with SYT1 normalized to the Syt $1^{+/+}$control. (E) Sample traces of EPSCs (right) and summary bar plot of the quantification of total EPSC charge transfer (left) from Syt1 $1^{-/}$neurons rescued with SYT1 normalized to the Syt1 $1^{+/+}$control. Artifacts and/or action potentials were blanked and substituted by arrows. (F) Bar plot of Pvr from Syt1 neurons rescued with SYT1 normalized to Syt1 $1^{+/+}$control. (G) Graph of the paired-pulse ratio. All data shown represent mean $+/$ - SEM. Statistical analysis was applied by Kruskal-Wallis test $\left({ }^{*} p=<\right.$ $0.05,{ }^{* *} \mathrm{p}=<0.01,{ }^{* * *} \mathrm{p}=<0.001,{ }^{* * * *} \mathrm{p}=<0.0001, \mathrm{~ns}=$ not significant). Scale bars $=5 \mu \mathrm{m}$.

Figure 3. Presynaptic SYT7 quantification of Synaptotagmin-7 in presence and absence of SYT1 and its silencing effect in Syt1 ${ }^{-/}$hippocampal glutamatergic neurons at different neuronal stages. (A) Representative images and (B) quantification of SYT1 (green) and SYT7 (blue) protein levels at VGLUT1 (red) positive regions at DIV11 and DIV16 of Syt1 ${ }^{+/+}$ and Syt $1 \%$ neurons. (C) Quantification of SYT1, SYT7 and VLGUT1 from Syt1\% autaptic neurons infected with lentivirus carrying either an empty vector or RNA interference (RNAI) for SYT7 at early and mature stages. Data are mean +/- SEM. Statistical significance and $p$ values were calculated using the Mann-Whitney $U$ test $\left({ }^{* *} P \leq 0.01,{ }^{* * *} P \leq 0.001,{ }^{* * *} P \leq 0.0001\right.$, ns $=$ not significant). Scale bars $=5 \mu \mathrm{m}$.

Figure 4. Knockdown of Synaptotagmin-7 in Syt1/- hippocampal glutamatergic neurons at different neuronal stages. (A) Representative traces at early (DIV11-12) and intermediate (DIV15-16) autaptic neuronal stages (right) and summary bar

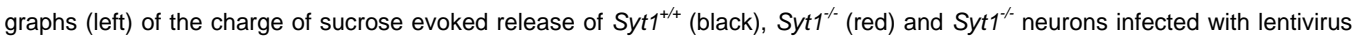
containing RNA interference (RNAi) for silencing SYT7 (purple) (B) Example traces of spontaneous release events (right) and summary bar graphs of spontaneous release rate (left) at the two different neuronal stages as A. (C) Representative EPSC traces (right) and summary bar graphs of total EPSC charge measured over an interval of 1s (left). (D) Summary bar graphs of vesicular release probability. Data are mean +/- SEM. Statistical significance and $p$ values were estimated by a Kruskal-Wallis test $\left({ }^{\star} \mathrm{P} \leq 0.05,{ }^{\star \star} \mathrm{P} \leq 0.01,{ }^{\star \star \star} \mathrm{P} \leq 0.001,{ }^{\star \star \star \star} \mathrm{P} \leq 0.0001, \mathrm{~ns}=\right.$ not significant).

Figure 5. Evaluation of SV priming and neurotransmitter release in Synaptotagmin-1 heterozygous neurons. All

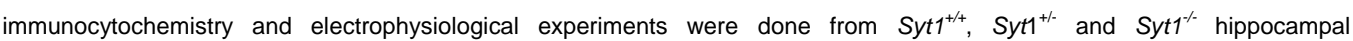




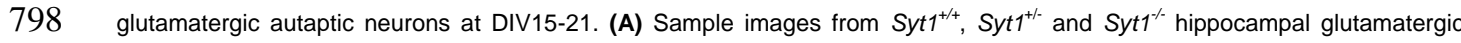
799 autaptic neurons double labeled with VGLUT1 (black puncta, left panel), SYT1 (black puncta middle panel) antibodies and

800 colocalization image of SYT1 in green and VLGUT1 in red (right merge panel), demonstrating the presence of Synaptotagmin-1 801 at the presynaptic terminals. Scale bar, $5 \mu \mathrm{m}$. (B) Bar plot of VGLUT1 average fluorescence intensities at presynaptic terminal 802 for the three Syt-1 genotypes (left), normalized to SYT1 expression levels of the Syt $1^{+/+}$neurons. Summary bar plot of SYT1 803 average fluorescence intensity at VGLUT1 positives puncta normalized to SYT1 average fluorescence intensity of Syt ${ }^{+1 /}$ (right). (C) Sample traces of sucrose responses (left) for the three Syt-1 genotypes. Bar graph of sucrose evoked current

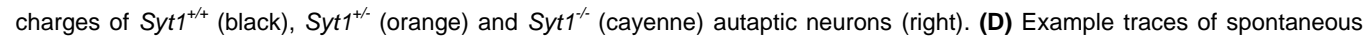
release events (left) and 106 mEPSC events superimposed from Syt $1^{+/+}, 198$ mEPSC events superimposed from Syt $1^{+/-}$and 257 mEPSC events superimposed from Syt $1^{-1}$ of a recording time period of 3 seconds in a $15 \mathrm{~ms}$ time window (center). Summary bar graph (right) of the spontaneous release rate of mEPSC events. (E) Representative EPSC traces (left) and summary bar graphs (right) of EPSC amplitudes. (F) Summary bar graph of total EPSC charge transfer. (G) Summary bar plot of the rise time (20-80\%) of EPSCs. (H) Bar graphs of the relative contribution of the synchronic and asynchronic components

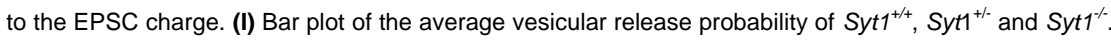

(J) Summary graph of the average pair-pulse ratio plotted against interpulse interval from 25 to $100 \mathrm{~ms}$ corresponding to 40,20 and $10 \mathrm{~Hz}$ of Syt $1^{+/ t}$ (black), Syt1 ${ }^{+/}$(orange) and Syt $1^{\text {\% }}$ (cayenne) autaptic neurons.

(K) Short-term plasticity response during high frequency $(20 \mathrm{~Hz})$ stimulation. Representative sample traces of EPSCs resulted from the stimulation of 20 consecutives APs separated by $50 \mathrm{~ms}$

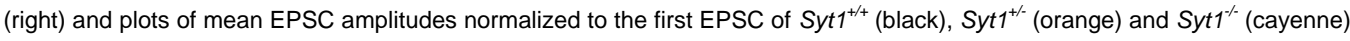
during train stimulation. Artifacts and action potentials were blanked from the traces and substituted by arrows in $\mathrm{E}$ and K. Data are mean $+/$ - SEM. Statistical significances and $p$ values were obtained by Kruskal-Wallis test $\left({ }^{*} P \leq 0.05,{ }^{* *} P \leq 0.01,{ }^{* *} P \leq 0.001\right.$, ${ }^{* * *} \mathrm{P} \leq 0.0001, \mathrm{~ns}=$ not significant). Scale bars $=5 \mu \mathrm{m}$.

Figure 6. Impact of Synaptotagmin-1 overexpression on synaptic properties. All immunocytochemistry and electrophysiological experiments were done from Syt1 ${ }^{+/+}$hippocampal glutamatergic autaptic neurons at DIV15-21. Representative images of $\mathrm{Syt}^{+/+}$autaptic neurons with and without overexpression of SYT1, co-immunolabeled for VGLUT1 (left), SYT1 (middle) and merge image (right), showing the presence of VGLUT1 in red and SYT1 in green. Scale bar, $5 \mu \mathrm{m}$. Quantification graphs of normalized VGLUT1 fluorescence intensities and SYT1/VGLUT1 fluorescence intensity ratios of Syt1 ${ }^{+/+}$ hippocampal neurons overexpressing GFP vector (black) or SYT1 (olive green) (right). (B) Representative sucrose-evoked current traces (left) and summary bar plot of RRP size (right). (C) Example traces of spontaneous release events (left) and summary bar graph (right) of spontaneous release rate. (D) Sample EPSC traces (left) and bar plot of EPSC amplitude (right). (E) Summary bar graph of average probability of vesicle release. (F) Bar graph of paired-pulse ratio (PPR) at $40 \mathrm{~Hz}$ (left). Representative sample traces of EPSCs resulted from the stimulation of 2 consecutives APs separated by $25 \mathrm{~ms}$ (right). Artifacts and/or action potentials are blanked and substituted by arrows in $\mathbf{D}$ and $\mathbf{F}$. All data shown represent mean $+/-$ SEM. Statistical analysis was applied Mann-Whitney $U$ test $\left({ }^{\star} \mathrm{P} \leq 0.05,{ }^{* *} \mathrm{P} \leq 0.01,{ }^{* \star *} \mathrm{P} \leq 0.001,{ }^{* \star * *} \mathrm{P} \leq 0.0001, \mathrm{~ns}=\right.$ not significant). Scale bars $=5 \mu \mathrm{m}$.

832 Figure 7. Dose dependence of Synaptotagmin-1 on neurotransmitter release functions. All immunocytochemistry and electrophysiological experiments were done from Syt1 ${ }^{+/+}$hippocampal glutamatergic autaptic neurons at DIV15-21. (A)

834 Representative images immunolabeled for VGLUT and SYT1 as figure 6, from Syt1 ${ }^{+/+}$hippocampal glutamatergic autaptic 
835 neurons infected with a lentiviral vector expressing a scramble RNA (control) or increasing amounts of a Syt1 RNA interference

836 (RNAi). Scale bar, $5 \mu \mathrm{m}$. Summary bar graphs of VGLUT1 fluorescence intensities at presynaptic terminal (left) and

837 SYT1/VGLUT1 fluorescence intensities ratios normalized to Syt $1^{+/+}$(right). (B) Plot of sucrose charges of Syt ${ }^{+/+}$control and

838 increasingly Knockdown SYT1 autaptic neurons normalized to the average Syt1 ${ }^{+/+}$control sucrose charge. (C) Summary bar

839 graph of spontaneous release rate normalized to control Syt1 $1^{+/+}$. (D) Normalized bar graphs of the effect on evoked EPSC

840 charge of titration of SYT1. (E) Bar graphs of the relative contribution of the synchronic and asynchronic components to the

841 EPSC total charge transfer with different amount of SYT1 expression levels. (F) Vesicular release probability (Pvr) normalized

842 to $S y t 1^{+/+}$. (G) Normalized summary graph of the pair-pulse stimulation at $40 \mathrm{~Hz}$. Statistical analysis was applied by Kruskal-

843 Wallis test $\left({ }^{*} \mathrm{P} \leq 0.05\right.$, ${ }^{* *} \mathrm{P} \leq 0.01$, ${ }^{* *} \mathrm{P} \leq 0.001$, ${ }^{* * *} \mathrm{P} \leq 0.0001$, ns = not significant). (H-K) Plots of RRP size (H), spontaneous

844 release rate $(\mathbf{I})$, EPSC charge $(\mathbf{J})$ and Pvr $(\mathbf{K})$ normalized to Syt1 ${ }^{+/+}$against the SYT1/VGLUT1 fluorescence intensity ratios,

845 obtained from the titration of SYT1 in autaptic neuronal cultures. To calculate SYT1 expression at synapses: Syt1 ${ }^{+/+}$(black),

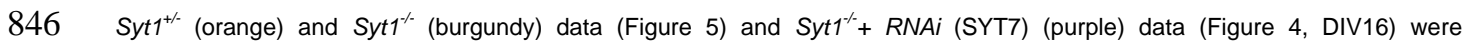

847 normalized to $S y t 1^{+/+}$and subtracting the Syt1 $1^{-/}$expression levels in the different experiments. For Syt1 $1^{+/+}$with the different

848 amounts of Syt1 RNAi (dark grey) and Syt1 $1^{+/+}$SYT1 (light grey) data (Figures 6 and 7) SYT1 expression levels were

849 normalized to $S y t 1^{+/+}$. All cells used for the model were from DIV15-21. Syt1 + RNAi (SYT7) cells were excluded from the

850 model, but the group is represented in the plot to illustrate its functional relevance. Discontinuous grey-line represent the curve-

851 fitting of the Hill function. All data shown represent mean $+/-\mathrm{SEM}$. $\mathrm{N}$ data. Scale bars $=5 \mu \mathrm{m}$. For $\mathbf{H}-\mathbf{K}$ data point values, see

852 also the table in Figure 7-1.

853 Figure 8. Impact of SYT1 RNAi viral titer on synaptic function in Synaptotagmin-1 ${ }^{+/}$neurons. All immunocytochemistry

854 and electrophysiological experiments were done from Syt1 ${ }^{+/}$hippocampal glutamatergic autaptic neurons at DIV15-21. (A)

855 Representative images of Syt $1^{+/}$cultured hippocampal glutamatergic neurons infected with a lentiviral vector expressing either

$856 R N A$ interference (RNAi) or scramble RNA as control. Scale bar, $5 \mu \mathrm{m}$. Summary bar graph of VGLUT1 (left) fluorescence at

857 synaptic terminal. Summary bar graph showing SYT1/VGLUT1 fluorescence at the synaptic terminal normalized to Syt $1^{+/+}$

858 (right). (B) Normalized bar graph of sucrose charge currents from autaptic cultures of Syt ${ }^{+/+}$neurons with $s c R N A$ (control) or

859 RNA interference. (C) Summary bar graph of spontaneous release rate. (D) Normalized bar graphs of the effect of RNA

860 interference on evoked EPSC charge. (E) Bar graphs of the relative contribution of the synchronic and asynchronic

861 components to the EPSC total charge transfer after a single AP with different amount of SYT1 levels. (F) Vesicular release

862 probability (Pvr) was calculated by the ratio of evoked EPSC charge and RRP size and normalized to Syt1 $1^{+/+}$. (G) Normalized

863 summary graph of the pair-pulse stimulation at $40 \mathrm{~Hz}$. Statistical analysis was applied by Kruskal-Wallis test $\left({ }^{\star} \mathrm{P} \leq 0.05\right.$, ${ }^{\star \star} \mathrm{P} \leq 0.01$,

$864{ }^{* \star *} \mathrm{P} \leq 0.001,{ }^{* \star *} \mathrm{P} \leq 0.0001, \mathrm{~ns}=$ not significant). All data shown represents mean $+/-\mathrm{SEM}$. Scale bars $=5 \mu \mathrm{m}$.

865 Table 1. Summary table with the electrophysiological parameters measured in autaptic hippocampal glutamatergic

866 neurons related to the culture stage. *PVr tendency towards an increased value, confirmed by STP experiment.

867 Figure 7-1. Values corresponding to Figure 7-1(H-K). 


\section{A \\ DIV11-12}

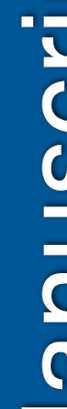

\section{DIV15-16}
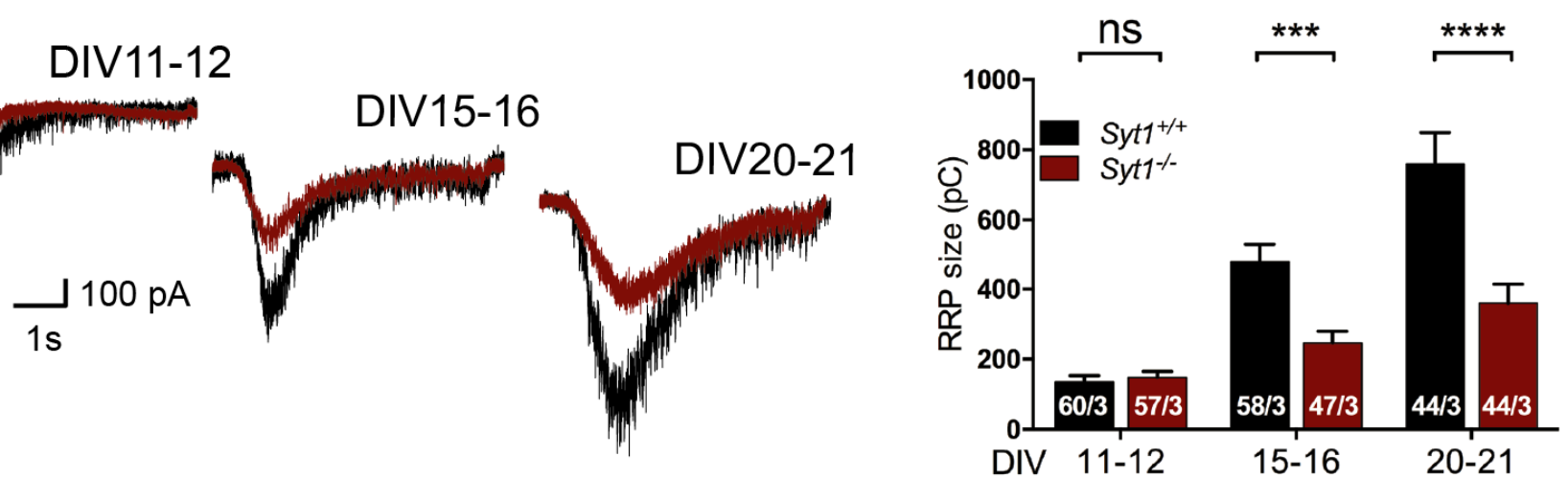

B

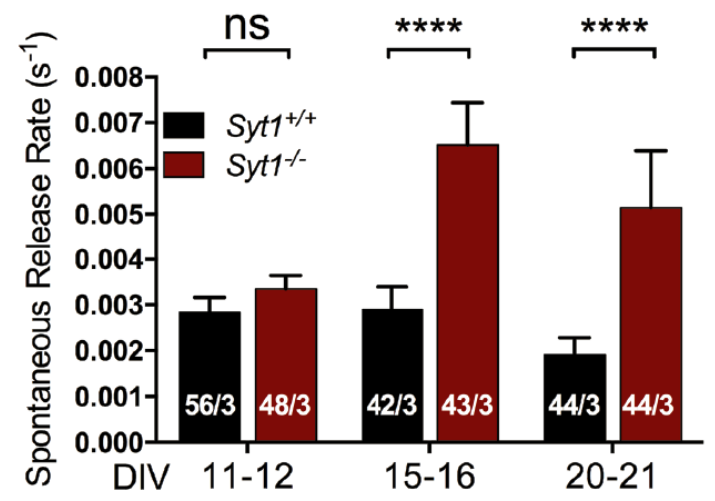

DIV11-12

(1)

$<$

0
0
0
2
2
2
2
2
C

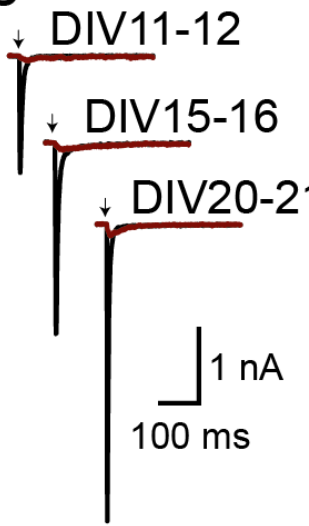

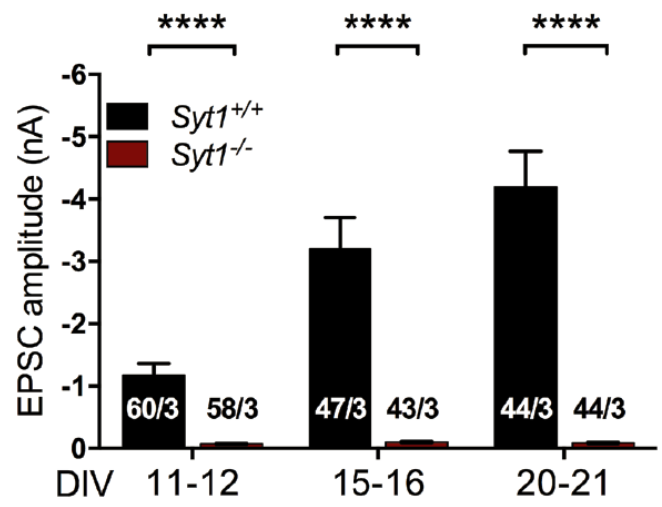

D

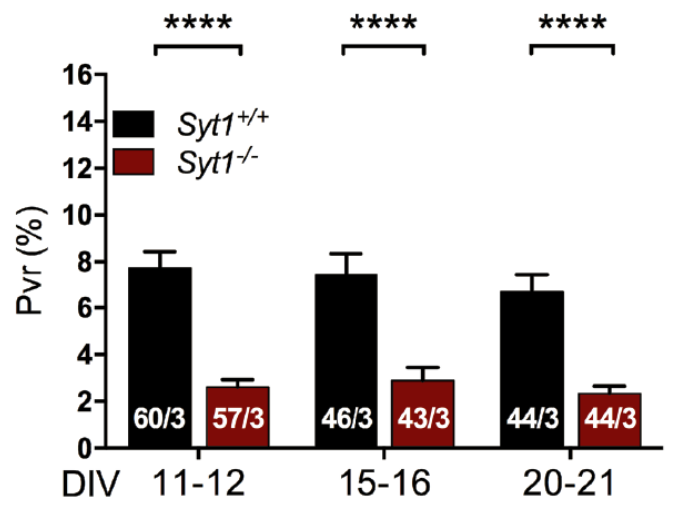


A

Syt1 ${ }^{+/+}$

Syt1\%-

B

$\stackrel{E}{2}$
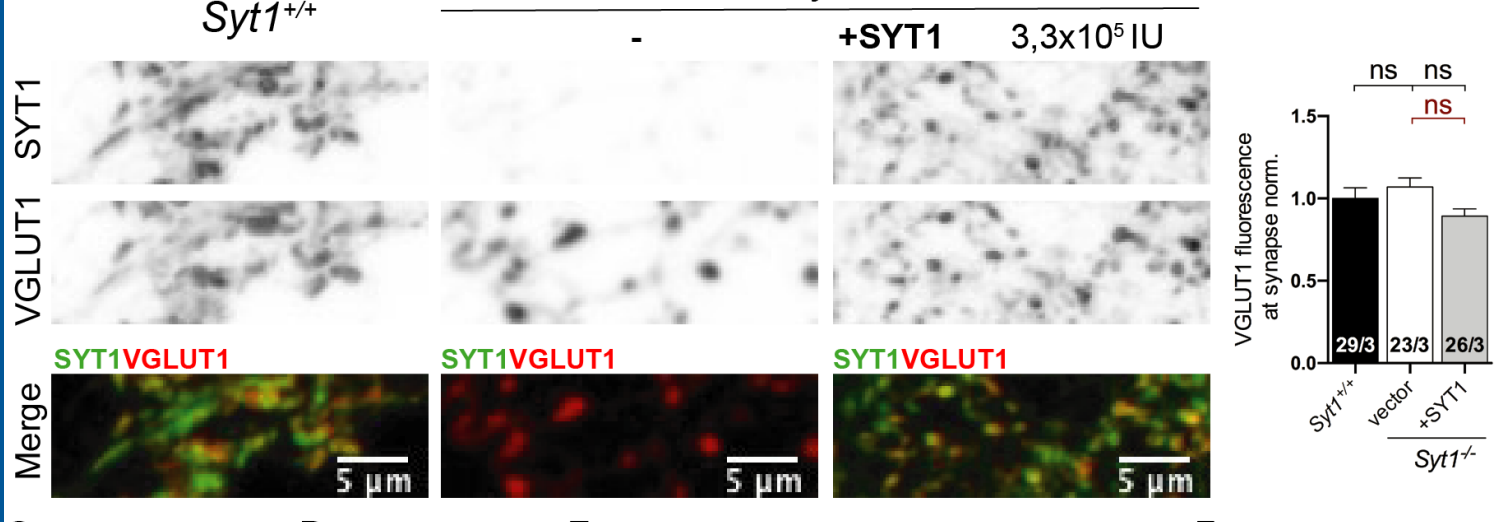

SYT1VGLUT1

SYT1VGLUT1

(1)
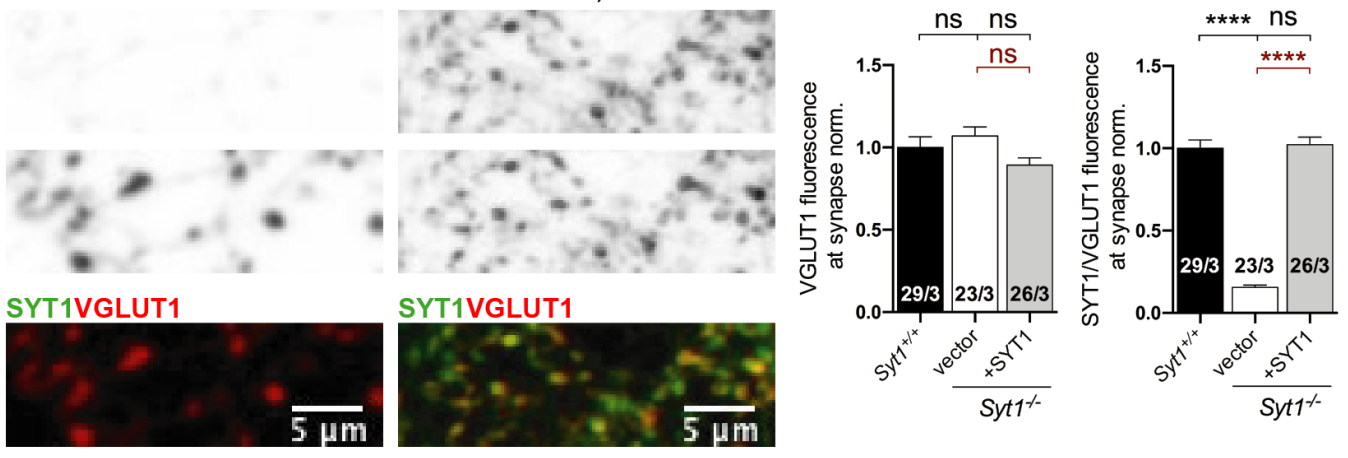

0
3

0

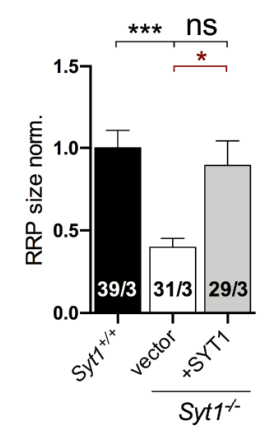

D

E

\section{$F$}

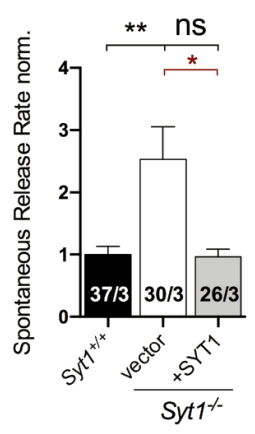

Syt1-1-

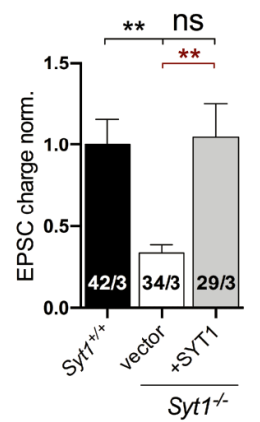

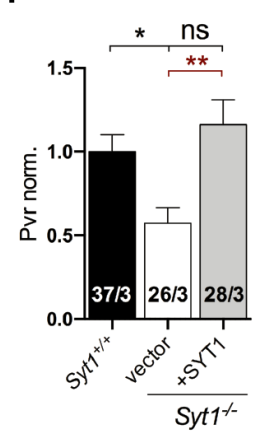

G

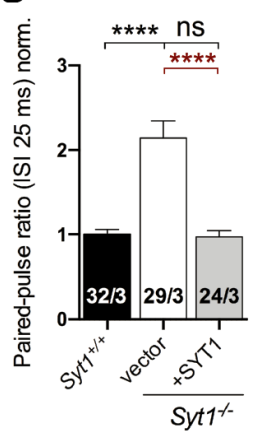



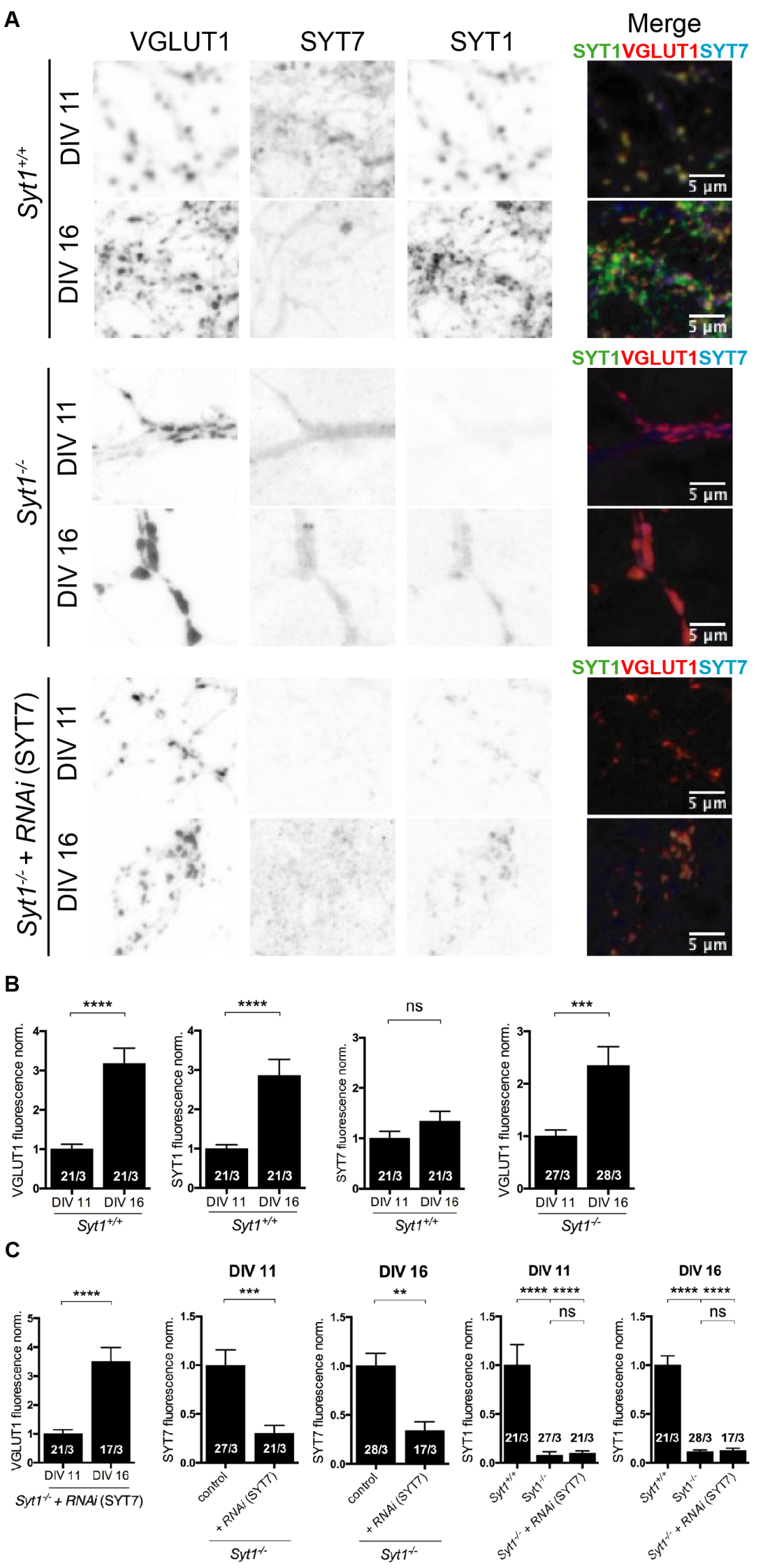
A

DIV11-12

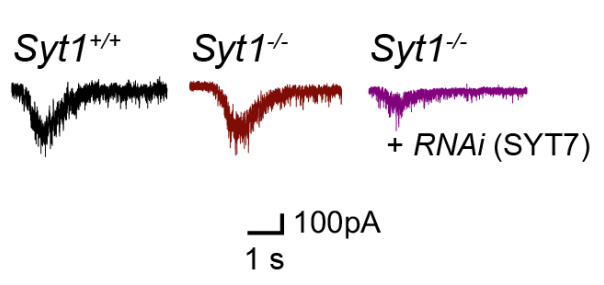

B

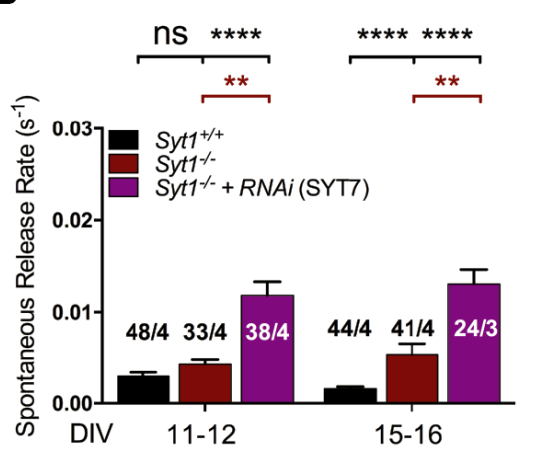

DIV15-16

\section{Syt1 $1^{+/+} \quad$ Syt1 $1^{--} \quad$ Syt1 $1^{-/}$}
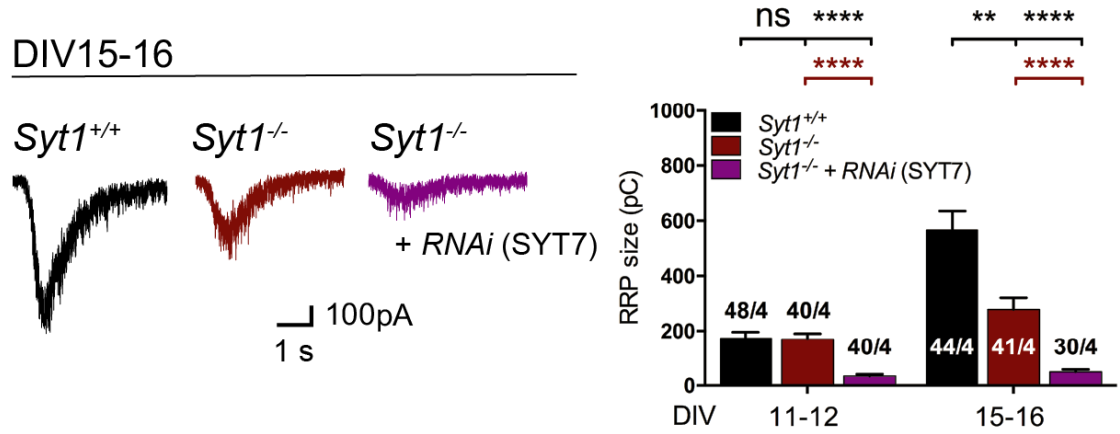

DIV11-12

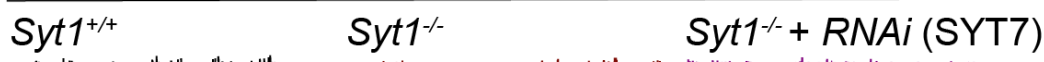

.

DIV15-16

Syt1 $1^{+/+} \quad$ Syt1 1 Syt1 $\quad$ SNAi (SYT7)

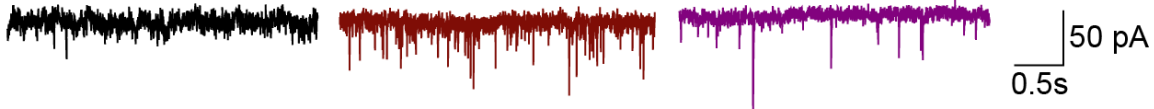

C
DIV11-12
$\frac{\text { DIV15-16 }}{\text { Syt1 } 1^{+/+}}$

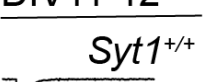

0

$\sqrt{S_{\text {Syt1 }}^{--}}$

Syt1--

+ RNAi (SYT7)

$\underbrace{}_{50 \mathrm{~ms}} 1 \mathrm{nA}$

syt1
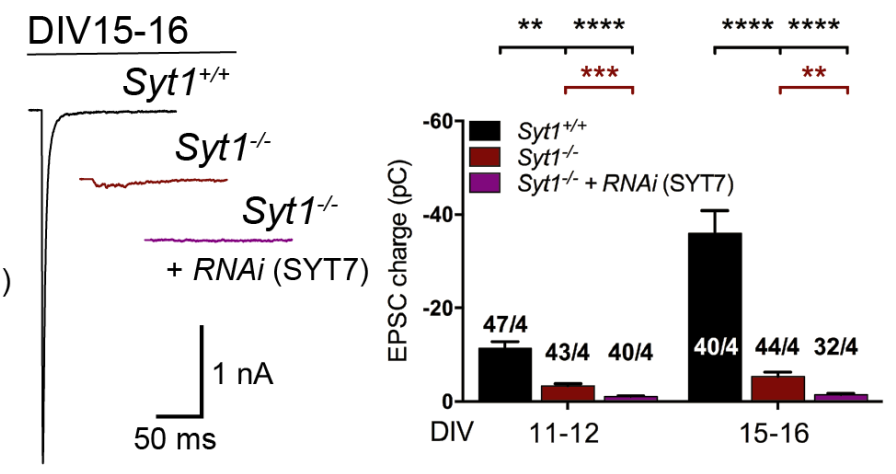

D
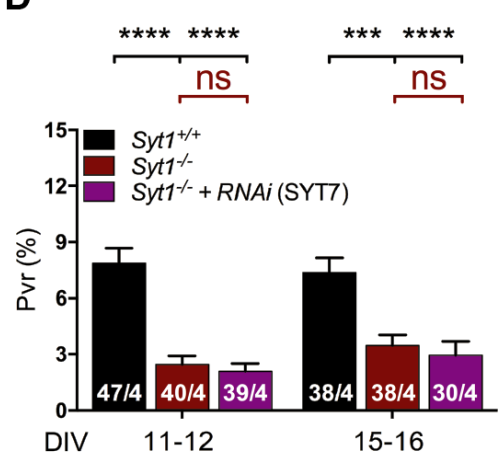
A
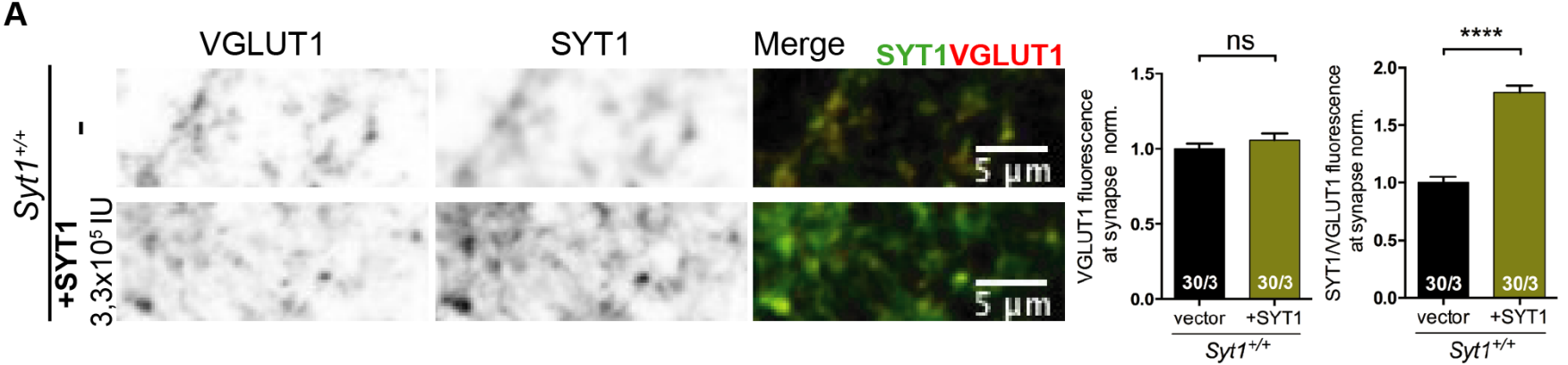

B

C
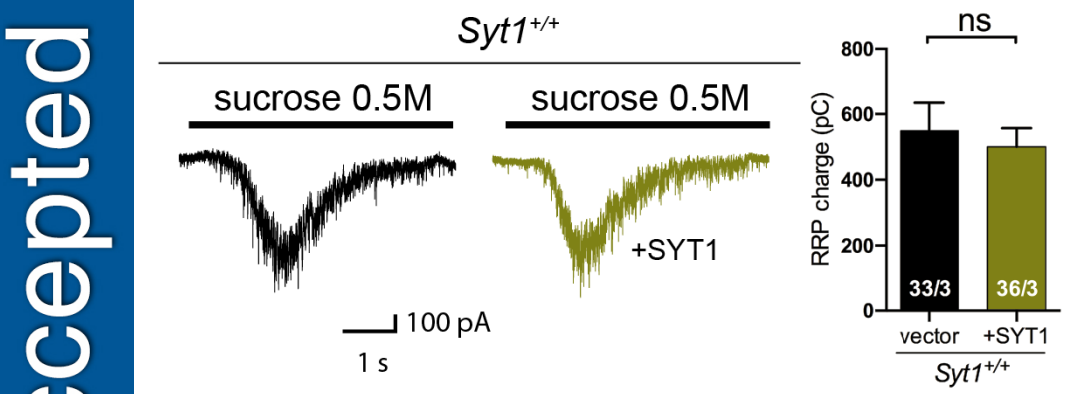

Syt $1^{+/+}$

Prim

0 D

E
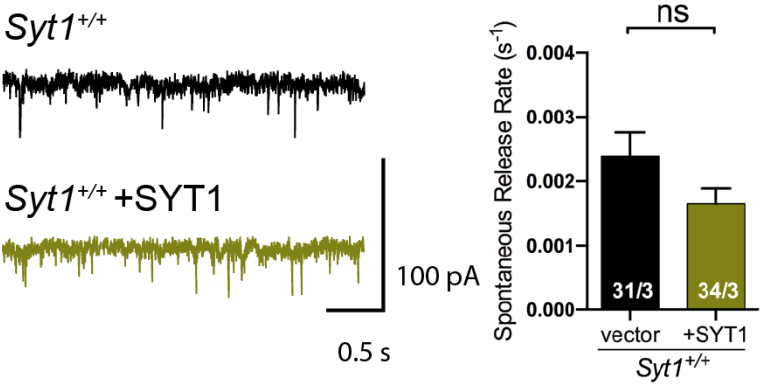

4
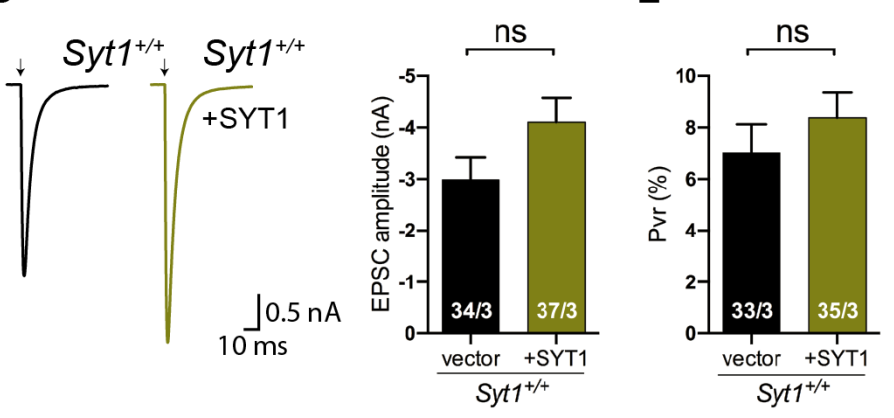

F
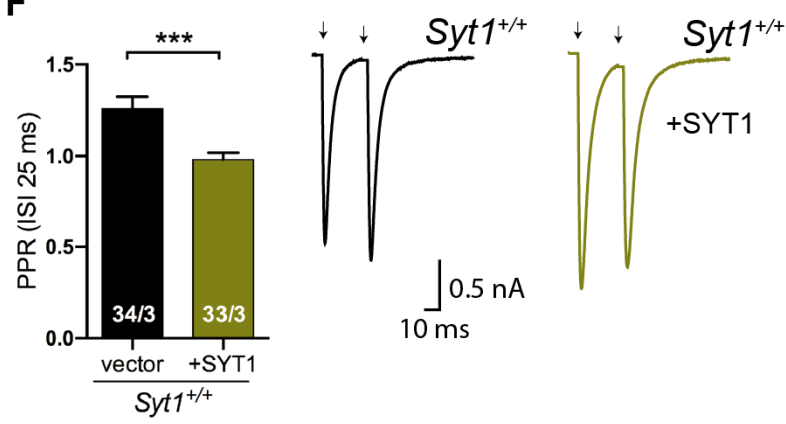
A

Syt $1^{+/+}$

Syt $1^{+/+}$

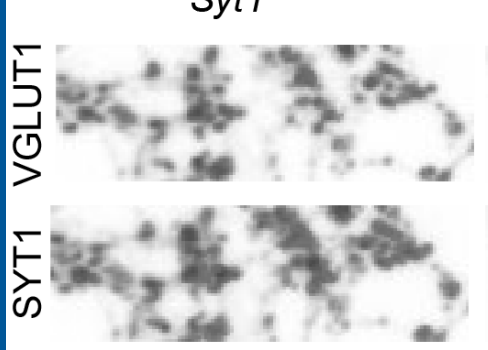

SYT1VGLUT1

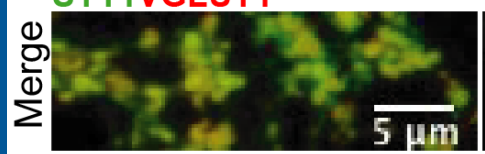

SYT1VGLUT1

$+R N A i 1,4 \times 10^{5} I \mathrm{IU}=\mathbf{1} \mathbf{x} \quad+R N A i 2,8 \times 10^{5} \mathrm{IU}=\mathbf{2} \mathbf{x}$

+ RNAi $5,7 \times 10^{5} I U=\mathbf{4} \mathbf{x}$

단

(1)

0

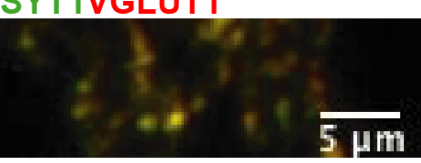

SYT1VGLUT1

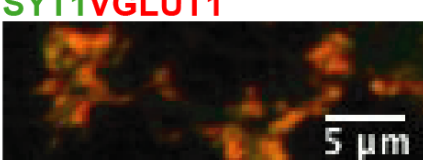

B
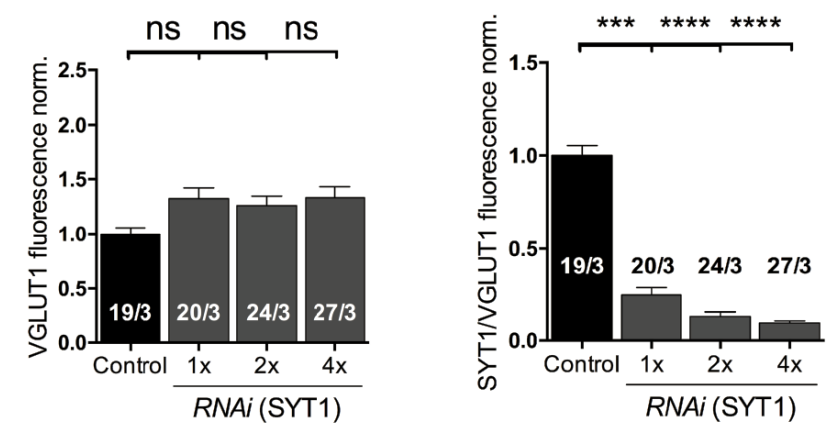

E

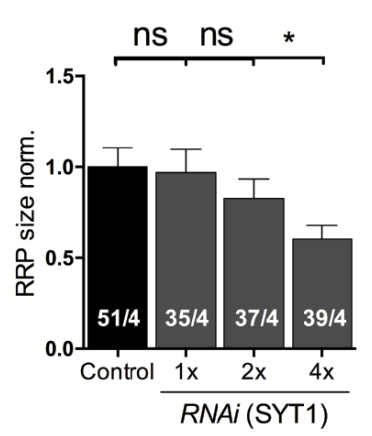

$\mathbf{F}$

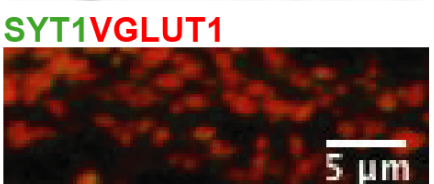

D

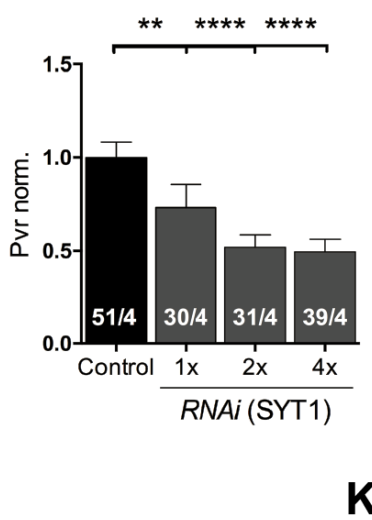

C
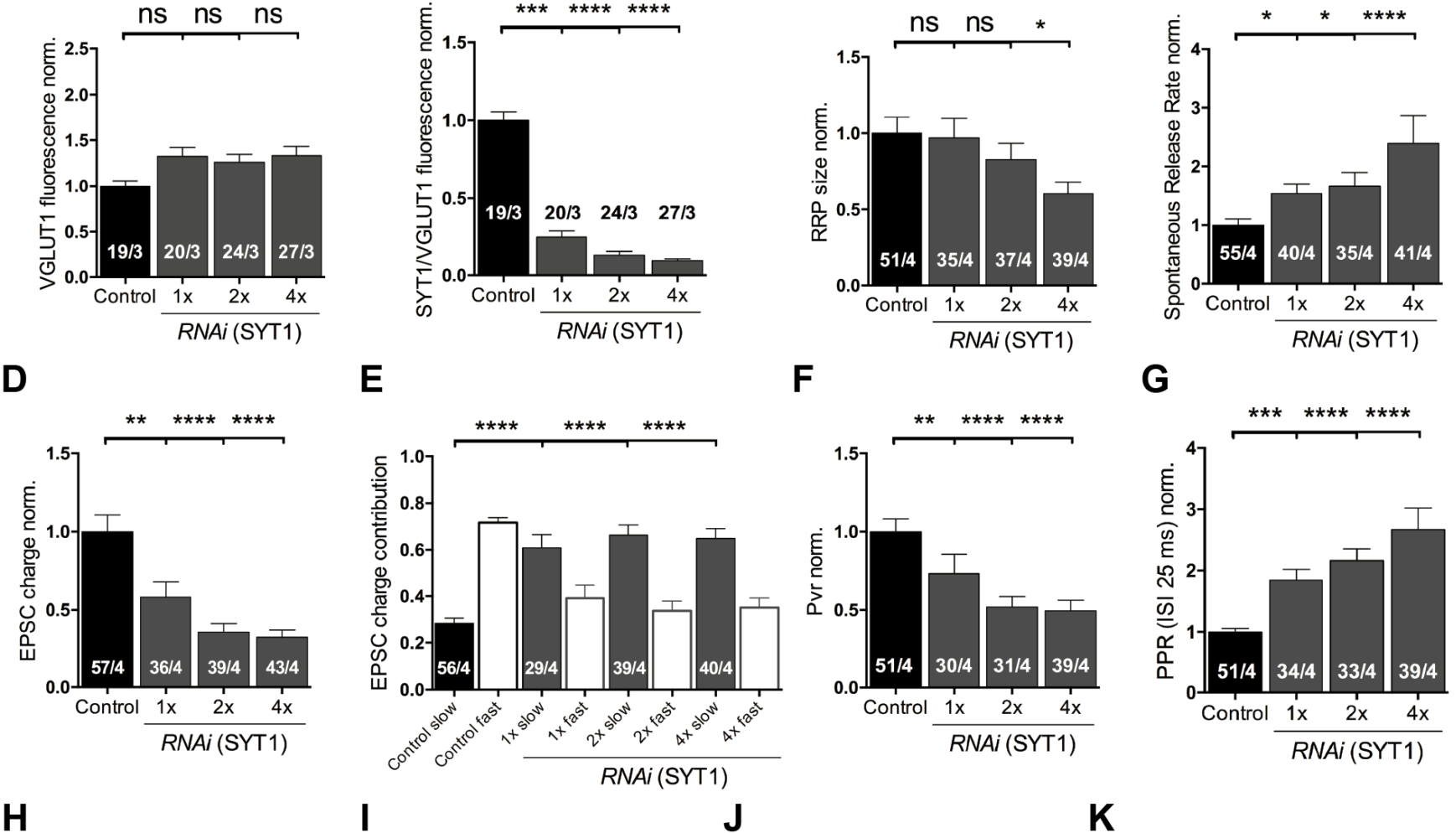

G

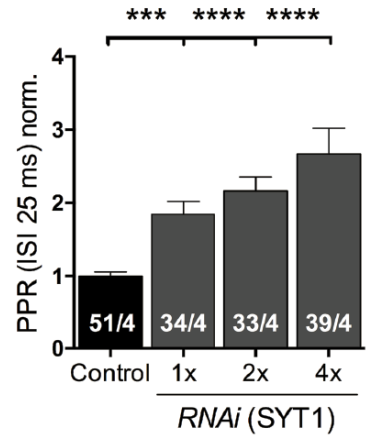

H

Not includ. in Model

K

Model data points

OSyt1 $^{-1-}+$ RNAi (SYTT)
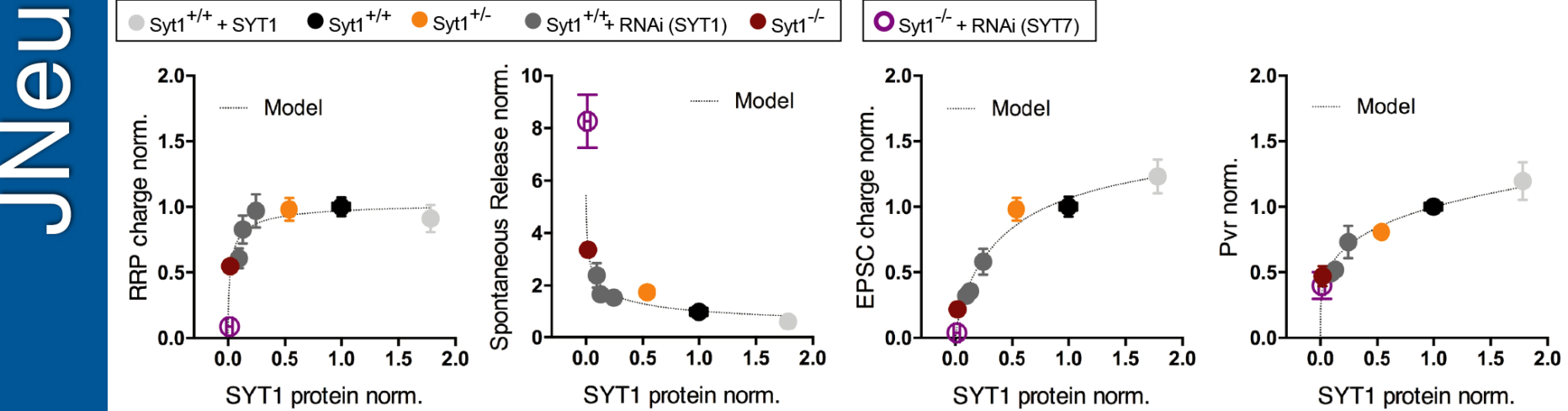
A

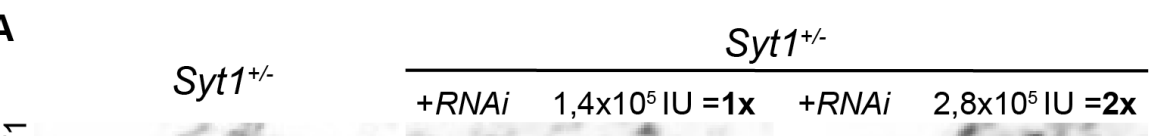

$\frac{c}{c}$
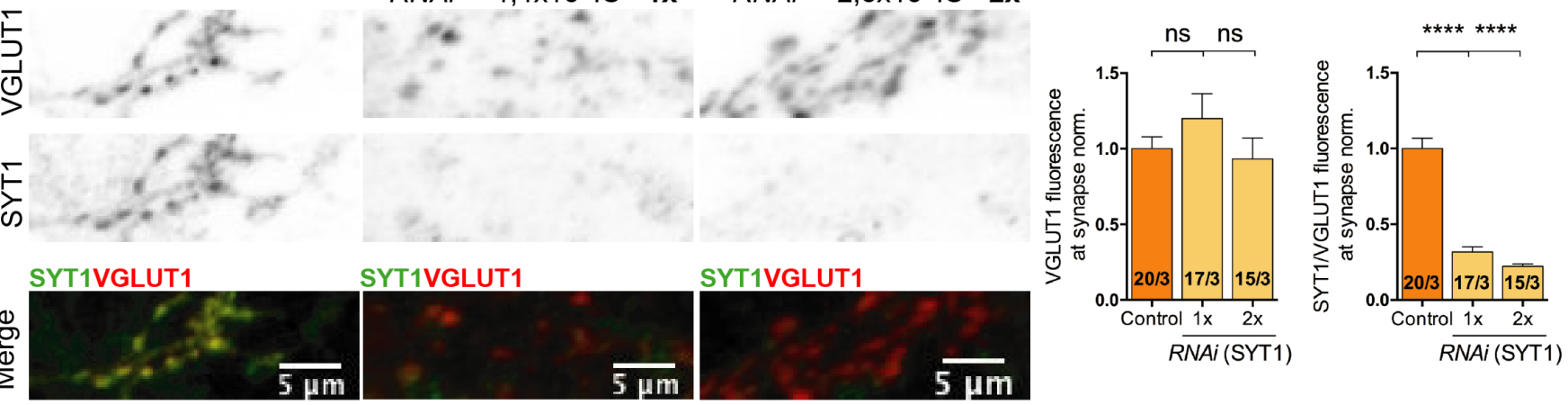

B

C

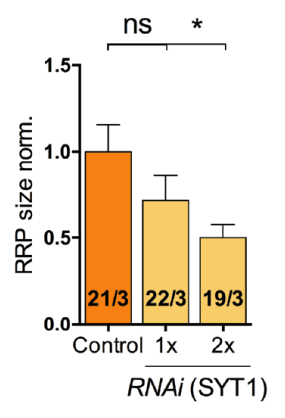

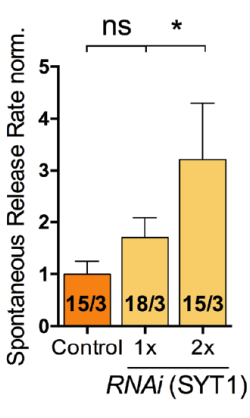

D

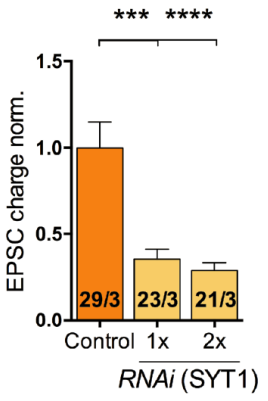

E

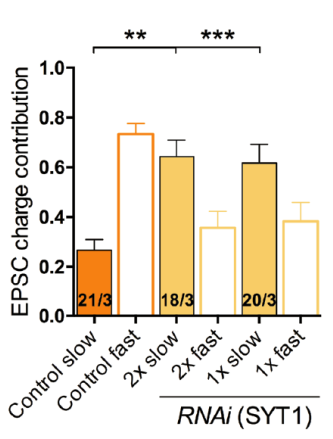

F

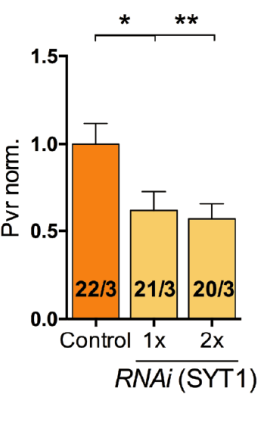

G

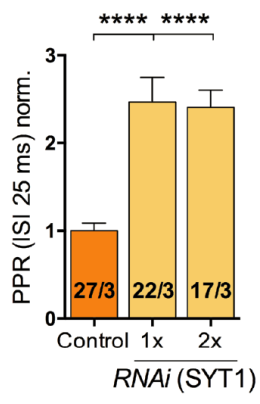


Table 1. Summary table with the electrophysiological parameters measured in autaptic hippocampal glutamatergic neurons related to the culture stage

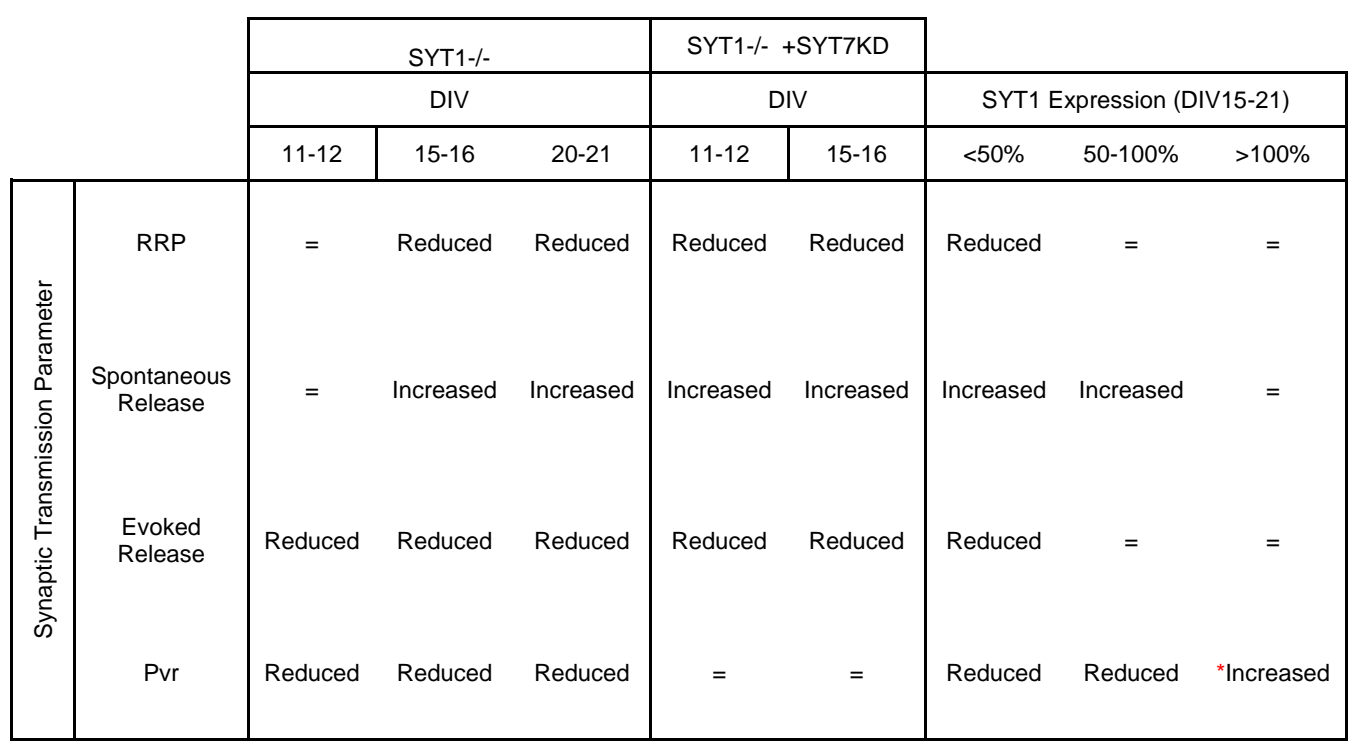

*Pvr tendency towards an increased value, confirmed by STP experiment 